ARTICLE

\title{
Non-canonical function of DGCR8 in DNA double- strand break repair signaling and tumor radioresistance
}

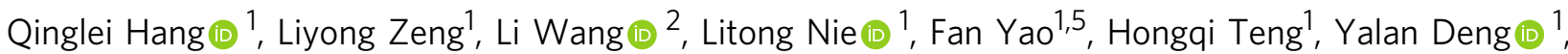
Shannon Yap ${ }^{1}$, Yutong Sun ${ }^{3}$, Steven J. Frank², Junjie Chen (1) ${ }^{1,4} \&$ Li Ma $\mathbb{B}^{1,4 凶}$

In response to DNA double-strand breaks (DSBs), repair proteins are recruited to the damaged sites. Ubiquitin signaling plays a critical role in coordinating protein recruitment during the DNA damage response. Here, we find that the microRNA biogenesis factor DGCR8 promotes tumor resistance to $\mathrm{X}$-ray radiation independently of its Drosha-binding ability. Upon radiation, the kinase ATM and the deubiquitinase USP51 mediate the activation and stabilization of DGCR8 through phosphorylation and deubiquitination. Specifically, radiation-induced ATM-dependent phosphorylation of DGCR8 at serine 677 facilitates USP51 to bind, deubiquitinate, and stabilize DGCR8, which leads to the recruitment of DGCR8 and DGCR8's binding partner RNF168 to MDC1 and RNF8 at DSBs. This, in turn, promotes ubiquitination of histone $\mathrm{H} 2 \mathrm{~A}$, repair of DSBs, and radioresistance. Altogether, these findings reveal the non-canonical function of DGCR8 in DSB repair and suggest that radiation treatment may result in therapy-induced tumor radioresistance through ATM- and USP51mediated activation and upregulation of DGCR8.

\footnotetext{
${ }^{1}$ Department of Experimental Radiation Oncology, The University of Texas MD Anderson Cancer Center, Houston, TX, USA. ${ }^{2}$ Department of Radiation Oncology, The University of Texas MD Anderson Cancer Center, Houston, TX, USA. ${ }^{3}$ Department of Molecular and Cellular Oncology, The University of Texas MD Anderson Cancer Center, Houston, TX, USA. ${ }^{4}$ The University of Texas MD Anderson UTHealth Graduate School of Biomedical Sciences, Houston, TX, USA. ${ }^{5}$ Present address: College of Biomedicine and Health, College of Life Science and Technology, Huazhong Agricultural University, Wuhan, Hubei, China. ${ }^{凶}$ email: Ima4@mdanderson.org
} 
$\mathrm{R}$ adiotherapy, the use of high-energy particles or waves such as X-rays to cure or shrink tumors, has been a common treatment for many patients with cancer ${ }^{1}$. Although both normal and cancerous cells are damaged by radiation, the goal of radiation therapy is to selectively destroy cancer cells, since normal cells can often repair much of the damage caused by radiotherapy ${ }^{2}$. Unfortunately, some tumors exhibit either intrinsic or therapy-induced radioresistance, and effective and safe radiosensitizers are still lacking ${ }^{3-6}$.

DNA double-strand breaks (DSBs), which are generated by endogenous agents and exogenous insults including ionizing radiation (IR), are highly cytotoxic cellular lesions that, if not properly repaired, can cause genomic instability, cell-cycle arrest, and cell death ${ }^{7-10}$. To maintain genome stability, cells have evolved a complex system called the DNA damage response (DDR) system, and radioresistant tumor cells often exhibit increased DDR and DNA repair ability ${ }^{3,10}$. In the DDR system, genomic DSBs are sensed by the MRE11-RAD50-NBS1 complex, which recruits the kinase ATM to the vicinity of DNA lesions. The resulting phosphorylation of the histone variant $\mathrm{H} 2 \mathrm{AX}$ by ATM allows the accumulation of MDC1 protein and its binding partners ${ }^{9,11-13}$. As the early event in a ubiquitination cascade, phosphorylated MDC1 recruits RNF8, a RING-type ubiquitin ligase that initiates mono-ubiquitination and K63-linked polyubiquitination of $\mathrm{H} 2 \mathrm{~A}$ type histones at sites of DNA damage ${ }^{14-17}$. Subsequently, another ubiquitin ligase, RNF168, is recruited to the chromatin surrounding DNA damage sites to augment the ubiquitination of histone $\mathrm{H} 2 \mathrm{~A} / \mathrm{H} 2 \mathrm{AX}$. An alternative model, however, proposes that RNF168 initiates H2A/H2AX ubiquitination on K13-15, while RNF8 extends the histone ubiquitination by adding K63-linked ubiquitin chains ${ }^{18}$. Ultimately, $\mathrm{H} 2 \mathrm{~A} / \mathrm{H} 2 \mathrm{AX}$ ubiquitination triggers a second wave of protein accumulation, including DNA repair proteins BRCA1 and 53BP14-17,19-23. Ubiquitination involved in DDR is subject to regulation at several levels, and dysregulated histone ubiquitination leads to aberrant transcriptional silencing, defects in DNA damage checkpoint activation and cell-cycle arrest, and genomic instability ${ }^{24}$. In addition to the ubiquitin ligases, several deubiquitinating enzymes (DUBs), such as OTUB1, USP3, USP16, USP44, and USP51, are also involved in DDR ${ }^{25-31}$. Altogether, these studies underscore the importance of coordinated ubiquitination at DNA damage sites during DDR. Despite these advances, how cells precisely orchestrate the ubiquitination and deubiquitination events and recruit the DNA repair proteins to the DNA lesions has not been fully unraveled.

MicroRNAs (miRNAs) are endogenously expressed small noncoding RNAs that regulate gene expression post-transcriptionally ${ }^{32}$. In the nucleus, the microprocessor complex consisting of the RNase Drosha and its binding partner DGCR8 cleaves the primary transcripts of miRNAs (pri-miRNAs) to generate miRNA precursors (pre-miRNAs). The pre-miRNAs are then exported by Exportin-5 to the cytoplasm, where they are cleaved by another RNase, Dicer, to generate mature miRNAs ${ }^{33}$. We and others have demonstrated that certain miRNAs participate in DDR and radiation respons $^{34-40}$. Moreover, the miRNA biogenesis machinery has been implicated in DNA repair outside of canonical miRNAmediated target mRNA cleavage and translational inhibition. For example, Dicer and Drosha can generate dsRNAs that promote DNA repair by facilitating the recruitment of DDR factors ${ }^{41-43}$ or through the formation of DNA:RNA hybrids around DNA break sites $^{44}$. Moreover, both Dicer and DGCR8 have been reported to regulate nucleotide excision repair of ultraviolet-induced lesions (pyrimidine dimers and base modifications) ${ }^{45,46}$. However, the precise function of miRNA biogenesis factors in DSB repair and radiotherapy response remains unknown. In this study, we uncovered a non-canonical function of DGCR8 in DSB repair signaling and tumor radioresistance. In response to IR, the kinase ATM and the deubiquitinase USP51 mediate the activation and stabilization of DGCR8 through phosphorylation and deubiquitination. Once phosphorylated, DGCR8 and its constitutive binding partner RNF168 in turn interact with MDC1 and RNF8 to promote histone ubiquitination, DSB repair, and radioresistance.

\section{Results}

DGCR8 promotes tumor radioresistance independently of Drosha binding. To examine the association between the key miRNA biogenesis proteins and radioresistance, we stably expressed Drosha, DGCR8, Exportin-5, or Dicer in LM2 cells (Fig. 1a), a lung-metastatic subline of MDA-MB-231 human breast cancer cells ${ }^{47}$. Only DGCR8, but not Drosha, Exportin-5, or Dicer, increased clonogenic survival after X-ray IR (Fig. 1b). Moreover, among these four proteins, only DGCR8 was substantially upregulated upon IR treatment in a time-dependent manner (Fig. 1c).

Next, we compared the effects of wild-type (WT) DGCR8 and the Drosha binding-deficient mutant $\triangle 692$-DGCR8, which lacks the Drosha-binding domain and RNA processing ability ${ }^{48}$, on clonogenic survival of several cell lines after Xray radiation treatment. Overexpression of the $\triangle 692$-DGCR8 mutant in LM2, T47D, BT549, HCT116, HepG2, and HeLa cell lines promoted radioresistance just like WT DGCR8 (Fig. 1d and Supplementary Fig. 1a). Conversely, shRNA-mediated knockdown of DGCR8 in LM2, MCF-7, and BT549 cell lines sensitized the cells to X-ray treatment (Supplementary Fig. 1b); this radiosensitizing effect could be reversed by ectopic expression of either WT DGCR8 or $\triangle 692$-DGCR8 (Supplementary Fig. 1c, d). Taken together, these results suggest that DGCR8 positively regulates cancer cell radioresistance independently of its Drosha-binding ability.

To determine whether DGCR8 is upregulated in radioresistant tumor cells, we used X-ray IR to select radioresistant subpopulations from LM2 and MCF-7 cell lines. After an 8-Gray (Gy) dose, surviving cells formed colonies. We pooled the colonies and repeated the dose twice (Fig. 1e). Cells derived from this selection, named LM2-R and MCF-7-R cells, showed increased clonogenic survival upon IR relative to the parental cells (Fig. If and Supplementary Fig. 1e). We also compared levels of $\gamma \mathrm{H} 2 \mathrm{AX}$, a marker of DSBs, based on the knowledge that IR-induced DSBs result in the formation of $\gamma \mathrm{H} 2 \mathrm{AX}$ foci, and that the persistence of $\gamma \mathrm{H} 2 \mathrm{AX}$ foci indicates delayed repair and is associated with radiosensitivity ${ }^{49-51}$. Notably, $12 \mathrm{~h}$ after irradiation, $\gamma \mathrm{H} 2 \mathrm{AX}$ levels stayed high in parental LM2 cells but decreased substantially in LM2-R cells (Fig. 1g), indicating that the LM2-R subline is more able to clear DSBs. We then examined the protein levels of Drosha, DGCR8, Exportin-5, and Dicer. Not only was DGCR8 markedly upregulated in LM2-R cells compared with parental LM2 cells, but it also showed a substantial increase after IR treatment (Fig. 1h). Similarly, DGCR8 protein was also upregulated in MCF-7-R cells compared with parental MCF-7 cells (Supplementary Fig. 1e). Because DGCR8 was upregulated in the radioresistant cells, we silenced its expression, which led to radiosensitization of both LM2-R (Fig. 1i, j) and MCF-7-R (Supplementary Fig. 1e) cells. Importantly, the inhibited clonogenic survival ability upon IR could be rescued by ectopic expression of either WT DGCR8 or the $\triangle 692$-DGCR8 mutant (Fig. 1i, j).

We further validated our findings in mice bearing LM2-R xenograft tumors. When the tumor diameters reached $8 \mathrm{~mm}$, we irradiated the tumors with a 2-Gy fractionated dose of X-ray once a day for 5 consecutive days a week, and the treatment lasted 2 weeks. As shown in Fig. 1k, knockdown of DGCR8, as well as restoration of DGCR8 expression, had no substantial effect on tumor growth without irradiation. In contrast, treatment with 
a

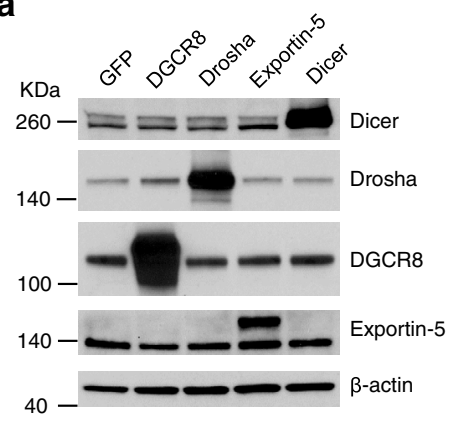

b

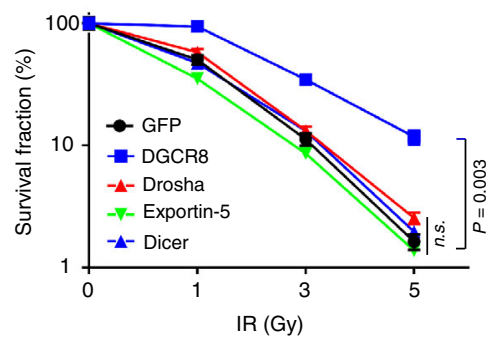

e
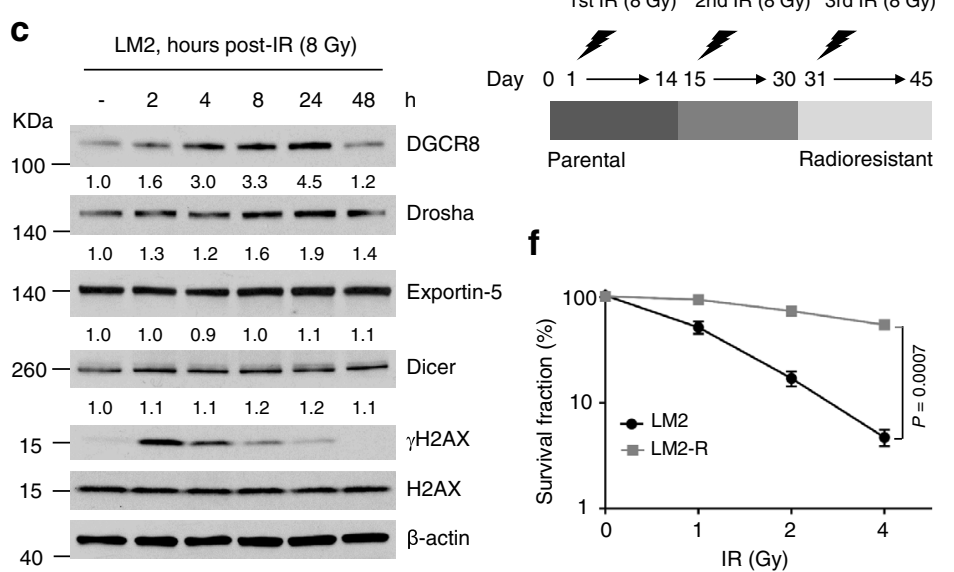

f

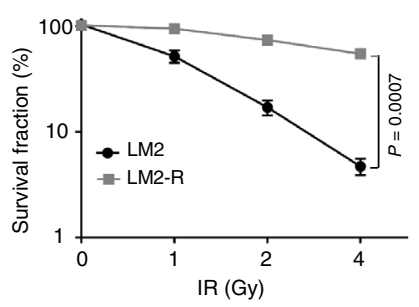

d

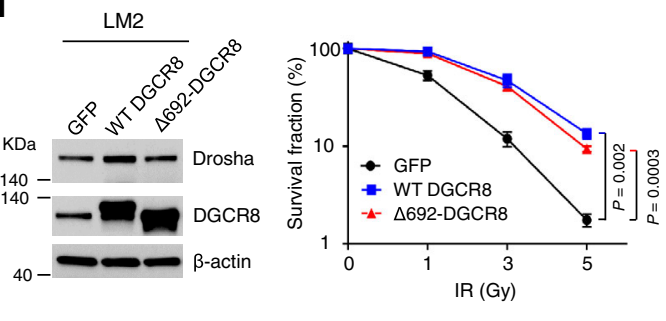

g

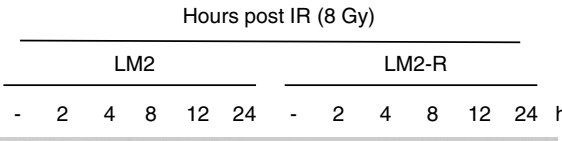

$15-\longrightarrow-1-1-1 \mathrm{H} 2 \mathrm{AX}$

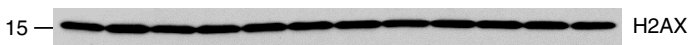

40- - - -1

\begin{tabular}{lllllllllll}
$\mathbf{h}$ & \multicolumn{3}{c}{ LM2 } & & \multicolumn{3}{c}{ LM2-R } \\
& - & 4 & 8 & 48 & & -4 & 8 & 48 & h post 8 Gy
\end{tabular}

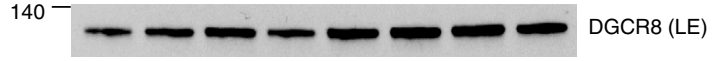

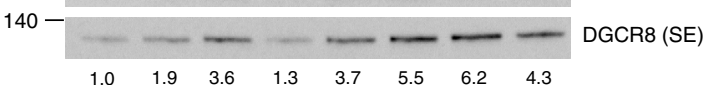

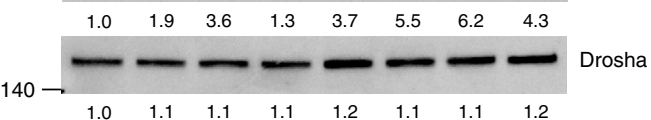

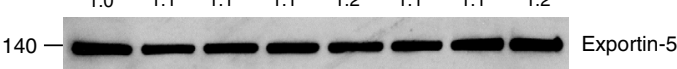

$\begin{array}{llllllll}1.0 & 0.9 & 1.0 & 1.0 & 0.9 & 0.9 & 1.0 & 1.1\end{array}$

$260-\square-----\square-$ Dicer

$\begin{array}{llllllll}1.0 & 1.4 & 1.7 & 1.5 & 1.5 & 1.4 & 1.2 & 0.9\end{array}$

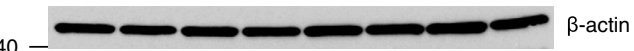

$\mathrm{KDa}$
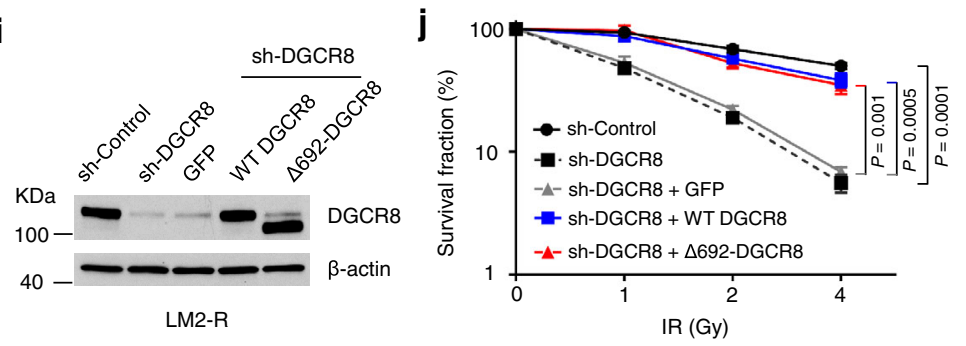

I

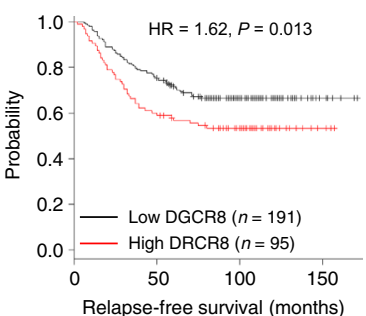

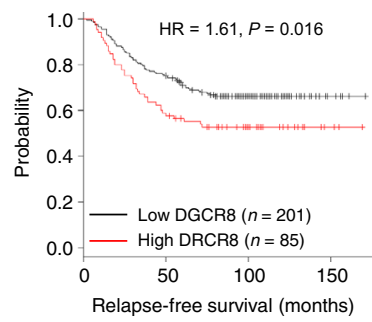

- sh-Control, no treatment

- sh-DGCR8, no treatment

- sh-DGCR8 + WT DGCR8, no treatment

- sh-DGCR8 + $\triangle 692-D G C R 8$, no treatment

- 2 sh-Control, XRT 2 Gy daily $\times 5$ times a week $\times 2$ weeks

-

- sh-DGCR8 + WT DGCR8, XRT 2 Gy daily $\times 5$ times a week $\times 2$ weeks

-๑) sh-DGCR8 $+\triangle 692-$ DGCR8, XRT 2 Gy daily $\times 5$ times a week $\times 2$ weeks fractionated X-ray led to sustained growth inhibition of tumors formed by DGCR8-knockdown cells, whereas tumors formed by either control LM2-R cells or cells with re-expression of DGCR8 (either WT or the $\Delta 692$ mutant) showed an initial response and then re-grew (Fig. 1k). Collectively, these findings indicate that DGCR8 promotes the radioresistance of breast cancer cells in vitro and in vivo independently of Drosha binding.

To assess the clinical relevance of DGCR8 expression to radiotherapy in patients, we analyzed a cohort of human breast cancer patients in which transcriptomic profiling was obtained from 286 tumor samples; $87 \%$ of these patients had received radiotherapy ${ }^{52}$. This analysis revealed that patients with high DGCR8 expression levels in their tumors had worse relapse-free survival than those with low DGCR8 expression levels (Fig. 11; with two different probes), suggesting that overexpression of DGCR8 may lead to radioresistance and eventually relapse in patients with breast cancer.

DGCR8 promotes the recruitment of RNF168 to RNF8 and MDC1 at DSBs, histone ubiquitination, and DNA repair after irradiation. To understand the molecular basis of DGCR8- 
Fig. 1 DGCR8 promotes tumor radioresistance independently of Drosha binding. a Immunoblotting of Dicer, Drosha, DGCR8, Exportin-5, and $\beta$-actin in LM2 cells transduced with GFP, Drosha, DGCR8, Exportin-5, or Dicer. b Clonogenic survival assays of LM2 cells transduced with GFP, Drosha, DGCR8, Exportin-5, or Dicer after X-ray ionizing radiation (IR) treatment. $n=3$ wells per group. $\mathbf{c}$ Immunoblotting of DGCR8, Drosha, Exportin-5, Dicer, $\gamma \mathrm{H} 2 \mathrm{AX}, \mathrm{H} 2 \mathrm{AX}$, and $\beta$-actin in LM2 cells collected at the indicated times after X-ray IR treatment. Quantification results were normalized to $\beta$-actin. d Immunoblotting of Drosha, DGCR8, and $\beta$-actin (left panel) and clonogenic survival assays (right panel) of LM2 stable cell lines overexpressing GFP, wild-type (WT) DGCR8, or $\Delta 692$-DGCR8 (the Drosha binding-deficient mutant). $n=3$ wells per group. e Schematic representation of the generation of radioresistant sublines (LM2-R and MCF-7-R) from parental LM2 and MCF-7 breast cancer cell lines by three rounds of X-ray irradiation. $\mathbf{f}$ Clonogenic survival assays of parental LM2 and LM2-R cells after $X$-ray IR treatment. $n=3$ wells per group. $\mathbf{g}$ Immunoblotting of $\gamma \mathrm{H} 2 \mathrm{AX}, \mathrm{H} 2 \mathrm{AX}$, and $\beta$-actin in parental LM2 and LM2-R cells collected at the indicated times after IR. $\mathbf{h}$ Immunoblotting of DGCR8, Drosha, Dicer, Exportin-5, and $\beta$-actin in LM2 and LM2-R cells collected at the indicated times after IR. LE long exposure, SE short exposure. Quantification results were normalized to $\beta$-actin. $\mathbf{i}$, $\mathbf{j}$ Immunoblotting of DGCR8 and $\beta$-actin (i) and clonogenic survival assays (j) of control or DGCR8-knockdown LM2-R cells transduced with GFP, WT DGCR8, or $\triangle 692-$ DGCR8. $n=3$ wells per group. $\mathbf{k}$ Tumor size of mice bearing xenograft tumors formed by control or DGCR8-knockdown LM2-R cells transduced with WT DGCR8 or $\triangle 692-D G C R 8$, with or without fractionated doses of localized X-ray IR treatment (XRT) using an X-RAD 320 irradiator. $n=5$ mice per group. I Kaplan-Meier curves of relapse-free survival of breast cancer patients (dataset: GSE2034; $n=286$ patients, $87 \%$ of whom received radiotherapy), stratified by DGCR8 expression levels. Data were generated from the KM Plotter (probes: 64474_g_at in the left panel and 91617_at in the right panel). The auto-select best cutoff was used in the analysis. Statistical significance was determined by a log-rank test. HR hazard ratio. Statistical significance in

$\mathbf{b}, \mathbf{d}, \mathbf{f}, \mathbf{j}$, and $\mathbf{k}$ was determined by a two-tailed unpaired $t$-test. Error bars are mean $\pm S E M$. Source data are provided as a Source Data file.

mediated radioresistance, we first examined $\gamma \mathrm{H} 2 \mathrm{AX}$ foci at different time points after X-ray IR, and we observed persistent $\gamma \mathrm{H} 2 \mathrm{AX}$ signals after irradiation in DGCR8-knockdown LM2 and BT549 cells, but not in the control cells (Fig. 2a, b). Importantly, DGCR8 was localized to IR-induced foci that overlapped with $\gamma \mathrm{H} 2 \mathrm{AX}$-positive foci in a time-dependent manner (Fig. 2a). Conversely, overexpression of either WT DGCR8 or $\Delta 692$ DGCR8 in parental LM2 cells accelerated the clearance of $\gamma \mathrm{H} 2 \mathrm{AX}$ after irradiation (Fig. 2c), suggesting that DGCR8 may empower cells to repair DSBs in an RNA processing-independent manner. Non-homologous end-joining (NHEJ) and homologous recombination (HR) are two distinct DSB repair pathways in eukaryotic cells $^{53,54}$. We used a previously established fluorescence-based reporter system that allows quantitative analysis of NHEJ and HR in the same cells through the repair of two inverted endonuclease I-SceI cuts ${ }^{55}$; by constructing a reporter cell line (LM2-DRR), we found that depletion of DGCR8 significantly decreased HR and NHEJ repair efficiency (Fig. 3a, b and Supplementary Fig. 2a).

To further investigate the molecular mechanism by which DGCR8 regulates DNA repair, we attempted to identify DGCR8interacting DDR proteins with or without IR treatment of LM2 cells by using a triple epitope (S-protein, FLAG tag, and streptavidinbinding peptide)-tagged version of DGCR8 (SFB-DGCR8). We performed tandem-affinity purification with streptavidin-sepharose beads and S-protein agarose beads, followed by mass spectrometric analysis, which identified several DGCR8-interacting DDR proteins in both non-irradiated and irradiated LM2 cells; among these DDR proteins, RNF168 was ranked the top one according to the intensitybased absolute quantitation (iBAQ) value (Supplementary Fig. 2b). On the other hand, another two DDR proteins, MDC1 and RNF8, were the top two DGCR8-interacting DDR proteins identified only in irradiated cells (Supplementary Fig. 2b). The cellular response to DSBs involves activation of ATM and subsequent production of $\gamma \mathrm{H} 2 \mathrm{AX}$, which binds and recruits MDC1 to DNA damage sites ${ }^{56,57}$. Then, the ubiquitin ligases RNF8 and RNF168 are recruited in an MDC1-dependent manner ${ }^{20,21,58}$. Consistent with our mass spectrometry results, co-immunoprecipitation (Co-IP) assays showed that IR treatment induced the interaction of DGCR8 with MDC1 and RNF8 in both LM2 and HEK293T cells, whereas the DGCR8RNF168 interaction was constitutive (Fig. $3 c$ and Supplementary Fig. 2c). By performing glutathione $S$ transferase (GST) pulldown assays under cell-free conditions, we detected interaction between purified DGCR8 and purified RNF168 (Supplementary Fig. 2d), suggesting that RNF168 is DGCR8's direct and constitutive binding partner. Importantly, by performing Co-IP experiments to examine the interactions between endogenous proteins, we found that knockdown of DGCR8 in LM2 cells blocked IR-induced RNF168RNF8 and RNF168-MDC1 interactions, without inhibiting the RNF8-MDC1 interaction (Fig. 3d), which suggests that DGCR8 is required for recruiting RNF168 to MDC1 and RNF8 after irradiation. Consistent with the DGCR8-knockdown effect (Fig. 3a, b), knockdown of RNF168 also reduced HR and NHEJ repair efficiency in LM2-DRR cells (Supplementary Fig. 2e-g).

Next, we performed chromatin extraction followed by IP, with or without MNase treatment. As shown in Fig. 3e, the recruitment of DGCR8, MDC1, RNF8, and RNF168 to chromatin was induced by irradiation, and the interactions of DGCR 8 with RNF168, RNF8, and MDC1 were detected in chromatin extracts. Interestingly, similar interaction patterns were observed with or without MNase treatment (Fig. 3e). These results indicate that the interactions of DGCR8 with RNF168, RNF8, and MDC1 occur on chromatin, but these protein-protein interactions are not mediated by chromatin. Importantly, immunofluorescent staining of irradiated LM2 cells showed that knockdown of DGCR8 did not affect the recruitment of MDC1 or RNF8 to DSBs, but abolished the localization of RNF168, 53BP1, and BRCA1 to DNA damage sites (Fig. 3f and Supplementary Fig. 3). Given that RNF8 and RNF168 ubiquitinate H2A to drive $\mathrm{DDR}^{16,18,20}$, we determined the effect of DGCR8 on RNF8- and RNF168-induced ubiquitination of H2A. Upon IR, both RNF8 and RNF168 could enhance mono-, di-, and poly-ubiquitination of $\mathrm{H} 2 \mathrm{~A}$, which was blocked by knockdown of DGCR8 (Fig. 3g). Taken together, these results suggest that after radiation treatment, DGCR8 mediates the recruitment of its binding partner RNF168 to MDC1 and RNF8 at DSBs, leading to amplification of histone ubiquitination.

Radiation-induced USP51 mediates deubiquitination and stabilization of DGCR8. We next sought to understand how radiation leads to the upregulation of DGCR8 protein. Although X-ray IR had little or no effect on DGCR8 mRNA levels (Supplementary Fig. 4a), irradiation of both LM2 and MCF-7 cells markedly upregulated DGCR8 protein to levels comparable to treatment with the proteasome inhibitor MG132 (Fig. 4a and Supplementary Fig. 4b). Moreover, we examined DGCR8 levels in the presence of the protein synthesis inhibitor cycloheximide ( $\mathrm{CHX}$ ) and found that compared with parental LM2 and MCF-7 cells, DGCR8 stability was much higher in the radioresistant sublines derived from X-ray irradiation (Fig. 4b and Supplementary Fig. 4c, d). We then examined proteolytic (K48-linked) and non-proteolytic (K63linked) ubiquitination levels of DGCR8. By using lysine mutants of ubiquitin (K48, K63, K48R, and K63R), we observed a substantial decrease in total and K48-linked ubiquitination of DGCR8 $24 \mathrm{~h}$ after 


\section{a}

Hours post
IR (2 Gy)
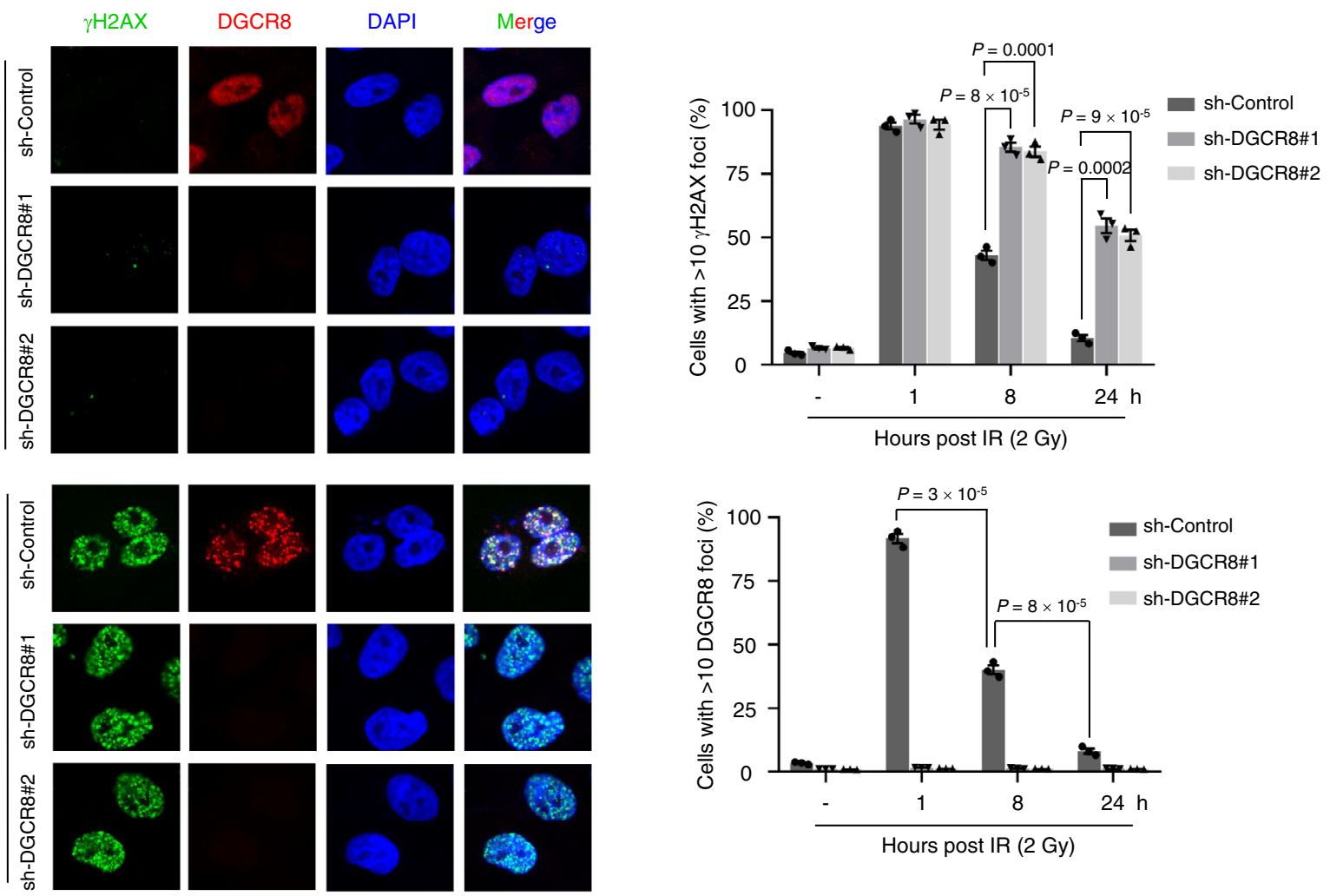

$1 \mathrm{~h}$
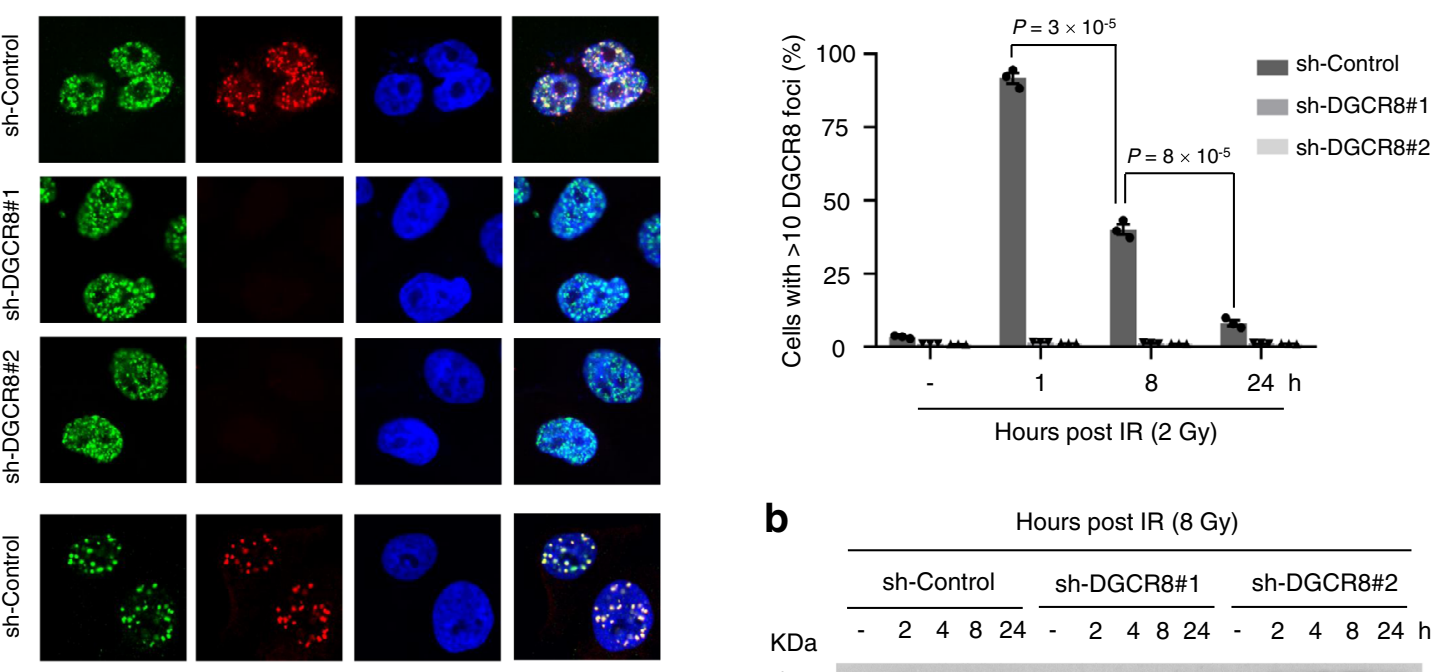

b
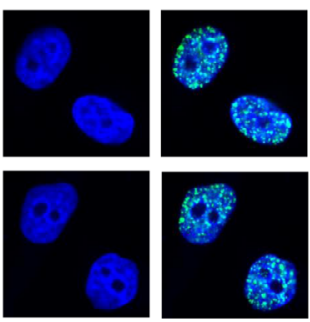

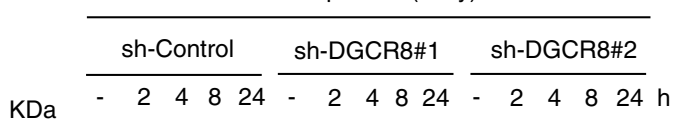
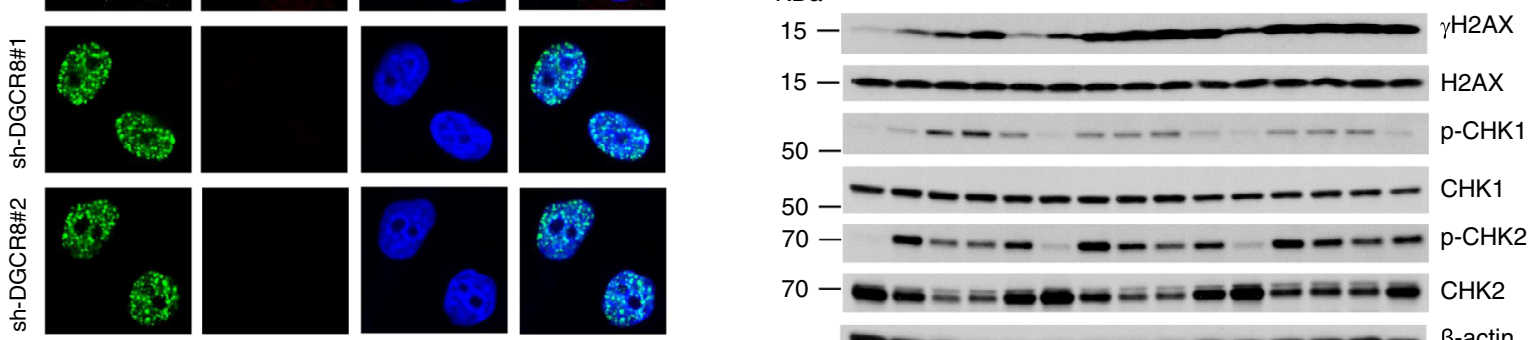

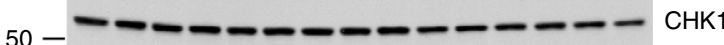

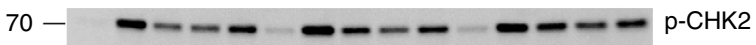

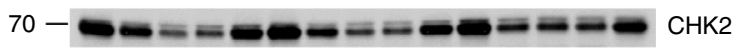
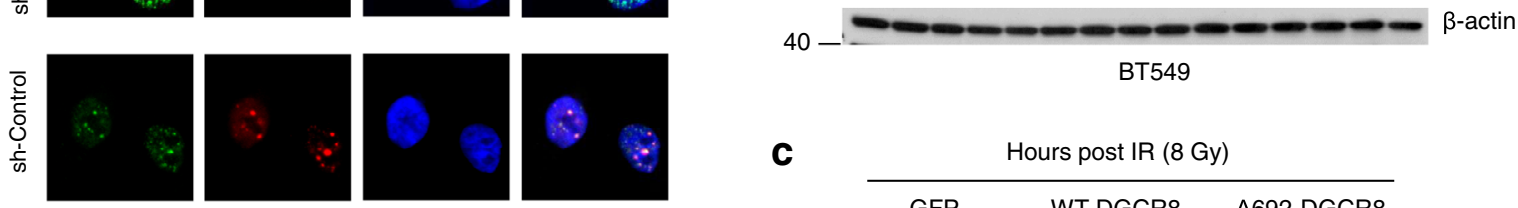

BT549

$24 \mathrm{~h}$
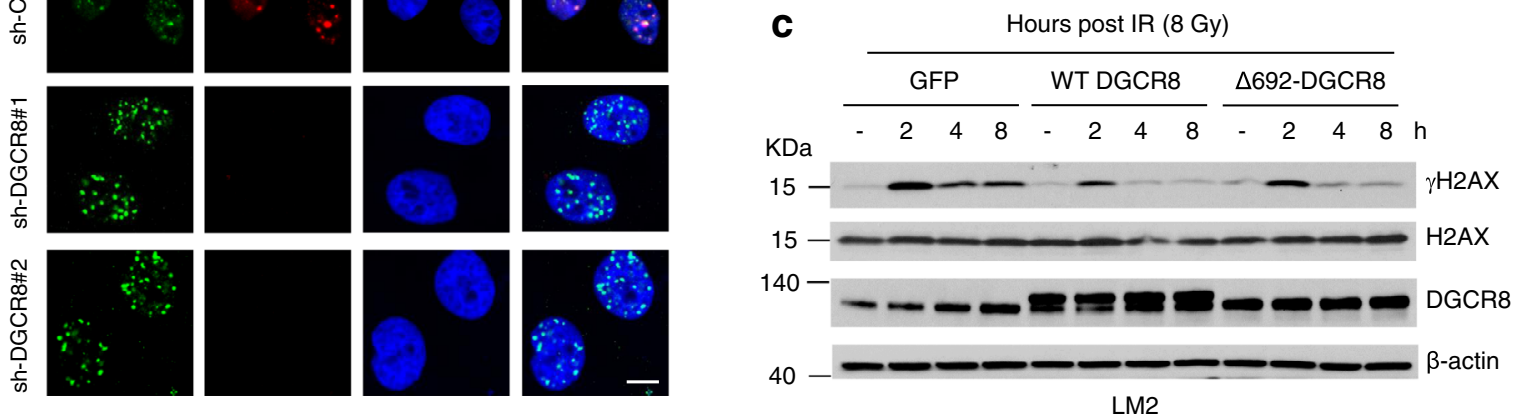

Fig. 2 DGCR8 promotes the clearance of DNA breaks after irradiation. a Left panels: DGCR8-knockdown LM2 cells were treated with 2-Gy ionizing radiation (IR). After the indicated times, cells were collected for immunofluorescent staining of $\gamma \mathrm{H} 2 \mathrm{AX}$ (green), DGCR8 (red), and DAPI (blue). Scale bar, $10 \mu \mathrm{m}$. Right panels: quantification of $\gamma \mathrm{H} 2 \mathrm{AX}$-positive and DGCR8-positive foci. $n=3$ biological replicates. Statistical significance was determined by a twotailed unpaired $t$-test. Error bars are mean \pm SEM. $\mathbf{b}$ Immunoblotting of $\gamma \mathrm{H} 2 \mathrm{AX}, \mathrm{H} 2 \mathrm{AX}, \mathrm{p}-\mathrm{CHK1}, \mathrm{CHK} 1, \mathrm{p}-\mathrm{CHK} 2, \mathrm{CHK} 2$, and $\beta$-actin in DGCR8-knockdown BT549 cells collected at the indicated times after X-ray IR treatment. c Immunoblotting of $\gamma \mathrm{H} 2 \mathrm{AX}, \mathrm{H} 2 \mathrm{AX}$, DGCR8, and $\beta$-actin in GFP-, WT DGCR8-, and $\triangle 692-D G C R 8$-overexpressing LM2 cells collected at the indicated times after X-ray IR treatment. Source data are provided as a Source Data file. 
a

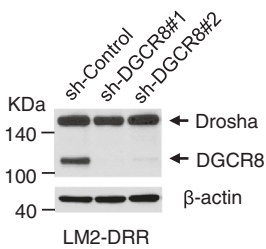

C
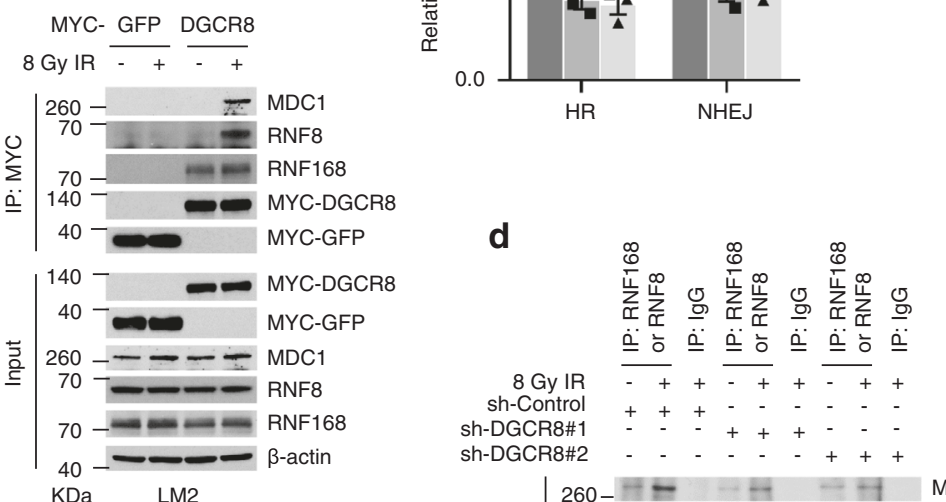

e
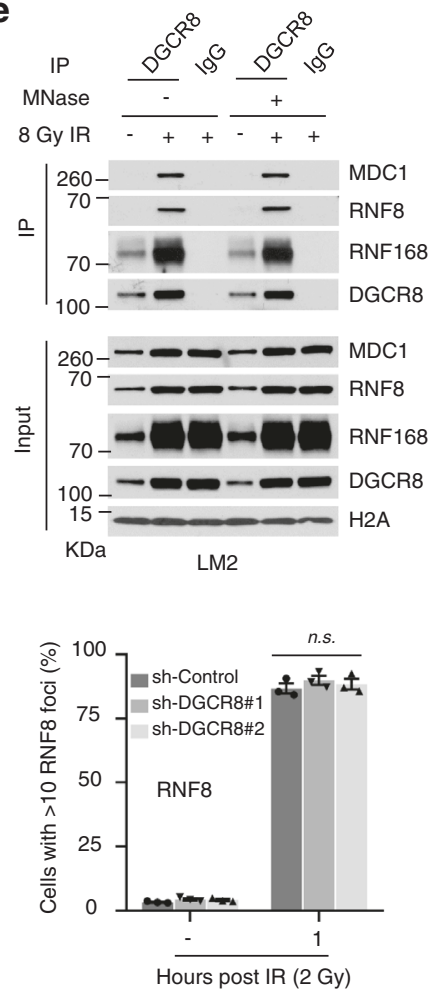

b

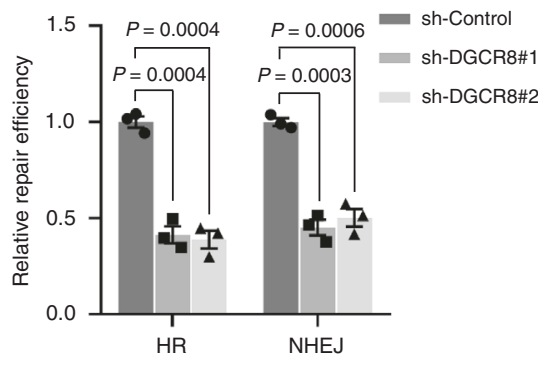

d

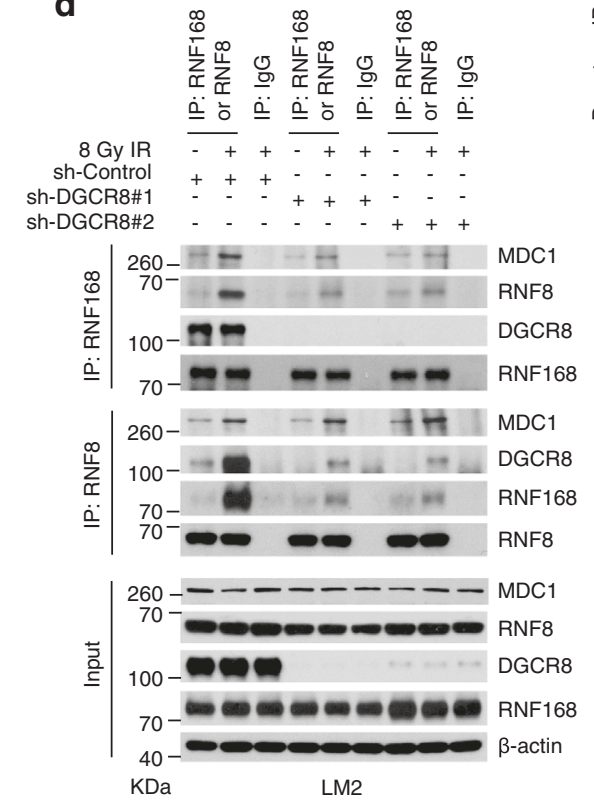

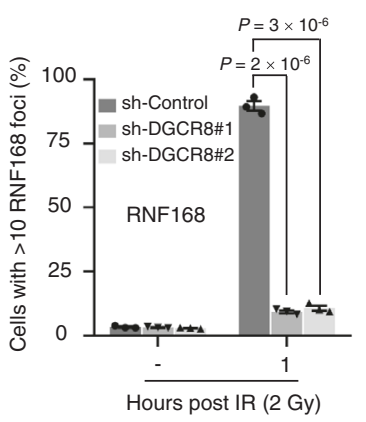

g

sh-DGCR8 sh-DGCR8

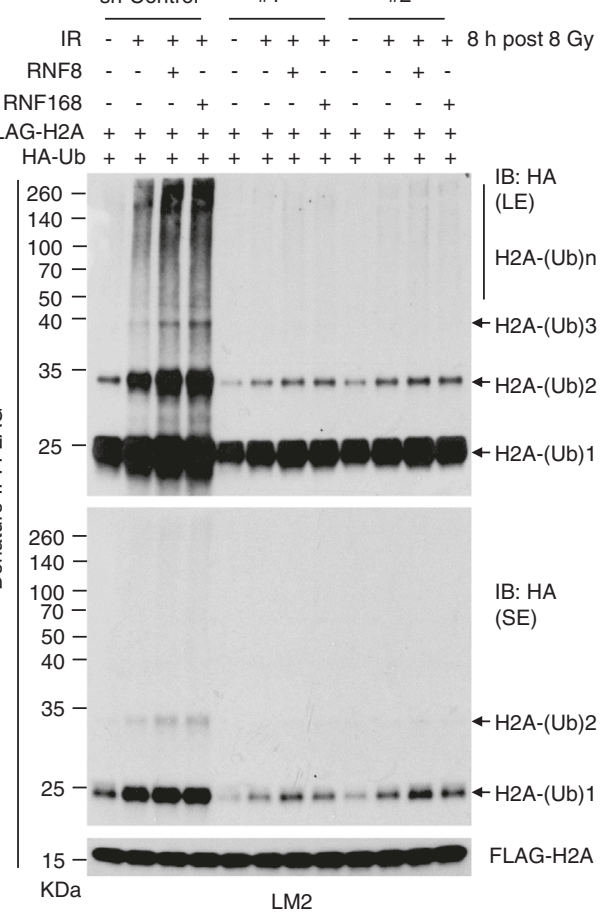

$\mathbf{f}$

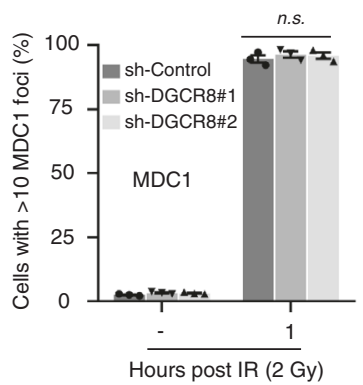

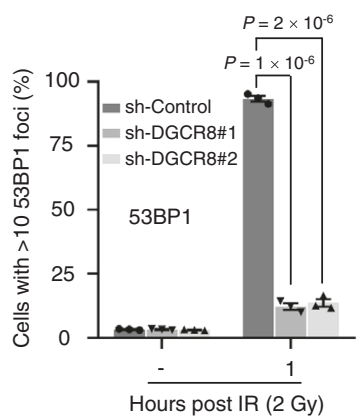

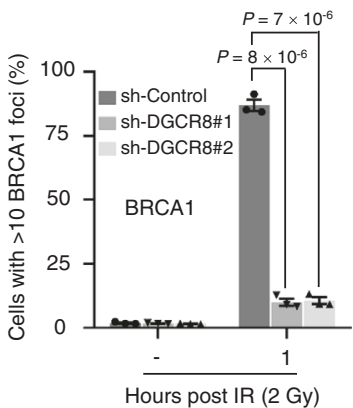

X-ray IR (Fig. 4c). These results suggest that radiation may stabilize DGCR8 protein by reducing its proteolytic ubiquitination.

DUBs are proteases that remove mono-ubiquitin or polyubiquitin chains from substrate proteins ${ }^{59}$. We screened for DGCR8-interacting DUBs by a pulldown assay using a panel of 68 SFB-tagged human DUBs ${ }^{60}$. We co-transfected MYC-DGCR8 and each SFB-tagged DUB into HEK293T cells, pulled down the DUBs with S-protein beads, and detected interaction of DGCR8 with six DUBs: USP10, USP36, USP39, USP43, USP49, and USP51 (Fig. 4d), which was verified by a confirmatory pulldown assay (Supplementary Fig. 4e). To determine the effect of these DUBs on DGCR8 ubiquitination, we co-expressed each SFB-tagged DUB with MYC-tagged DGCR8 and HA-tagged ubiquitin in HEK293T cells, and immunoprecipitation assays under denaturing conditions revealed that ectopic expression of either USP36 or USP51 reduced the poly-ubiquitination of DGCR8 (Fig. 4e). Through overexpression and knockdown of USP36 or USP51, we observed that both DUBs positively regulated the levels of endogenous DGCR8 protein in LM2 (Fig. 4f) and HEK293T (Supplementary Fig. 4f) cells. Moreover, Co-IP assays confirmed that both USP36 and USP51 could be pulled down by DGCR8 (Fig. 4g), and that both DUBs interacted with DGCR8 at endogenous levels in LM2 cells (Fig. 4h).

We then examined the subcellular localization of USP36, USP51, and DGCR8. Consistent with previous reports $31,48,61,62$, we detected USP36 specifically in the nucleolus, whereas both USP51 and DGCR8 proteins were localized diffusely in the nucleus 
Fig. 3 DGCR8 promotes the recruitment of RNF168 to RNF8 and MDC1 at DSBs, histone ubiquitination, and DNA repair upon irradiation.

a Immunoblotting of Drosha, DGCR8, and $\beta$-actin in the LM2-DRR (expressing the pLCN DSB Repair Reporter) cell line transduced with DGCR8 shRNA. b Knockdown of DGCR8 decreased HR and NHEJ efficiency in LM2-DRR cells. Two days after co-transfection of I-Scel endonuclease and an exogenous donor for HR (pCAGGS DRR mCherry Donor EF1a BFP) into the DGCR8-knockdown LM2-DRR cells, the percentages of GFP-positive and mCherry-positive cells, gated on BFP-positive cells, were determined by flow cytometry. Repair by HR or NHEJ leads to mCherry or GFP expression. Data were normalized to the control cells. $n=3$ biological replicates. c MYC-DGCR8-overexpressing LM2 cells were treated with IR ( 8 Gy) and cultured for $1 \mathrm{~h}$, followed by pulldown with MYC beads and immunoblotting with the indicated antibodies. $\mathbf{d}$ Control and DGCR8-knockdown LM2 cells were treated with IR (8 Gy) and cultured for $1 \mathrm{~h}$, followed by immunoprecipitation with an antibody against RNF168 or RNF8 and immunoblotting with the indicated antibodies. e Chromatin was extracted from LM2 cells that were treated with IR ( $8 \mathrm{~Gy}$ ) and cultured for $1 \mathrm{~h}$. The chromatin fractions, with or without MNase treatment, were immunoprecipitated with a DGCR8-specific antibody and immunoblotted with the indicated antibodies. f Quantification of MDC1, RNF8, RNF168, 53BP1, and BRCA1 foci in DGCR8-knockdown LM2 cells. Cells were incubated for $1 \mathrm{~h}$ after 2-Gy IR and immunostained with antibodies against $\gamma \mathrm{H} 2 \mathrm{AX}$, MDC1, RNF8, RNF168, 53BP1, and BRCA1 (see representative images in Supplementary Fig. 3). $n=3$ biological replicates. g Control and DGCR8-knockdown LM2 cells with stable overexpression of FLAG-H2A and RNF8 or RNF168 were transfected with HA-ubiquitin (Ub), treated with IR ( 8 Gy), and cultured for 8 h, followed by immunoprecipitation with anti-FLAG beads and immunoblotting with antibodies against HA and FLAG. Before immunoprecipitation, lysates were heated at $95^{\circ} \mathrm{C}$ for $5 \mathrm{~min}$ in the presence of $1 \%$ SDS (for denaturing), followed by a 10 -fold dilution with lysis buffer and sonication. LE long exposure, SE short exposure. Statistical significance in $\mathbf{b}$ and $\mathbf{f}$ was determined by a two-tailed unpaired $t$-test. Error bars are mean \pm SEM. n.s. not statistically significant. Source data are provided as a Source Data file.

(Supplementary Fig. 4g, h). To validate the direct interaction between USP51 and DGCR8, we expressed and purified SFB-USP51 and MBP-DGCR8 proteins, and in vitro pulldown assays demonstrated that USP51 bound to DGCR8 (Fig. 4i), indicating that DGCR8 may be directly regulated by USP51. To determine whether USP51 regulates the stability of DGCR8, we performed CHX treatment of the radioresistant LM2 (LM2-R) cells, finding that DGCR8 was less stable upon knockdown of USP51 (Fig. 4j).

To determine the deubiquitinating ability of USP51 towards DGCR8, we generated the catalytically inactive mutant of USP51, $\mathrm{C}_{372 S^{63}}$. Overexpression of WT USP51, but not the C372S mutant, removed K48-linked but not K63-linked poly-ubiquitin chains of DGCR8 in HEK293T cells (Supplementary Fig. 4i). To determine whether USP51 directly deubiquitinates DGCR8, we incubated purified USP51 and ubiquitinated DGCR8 purified from HEK293T cells in a cell-free system. Purified USP51, but not the C372S mutant, strongly deubiquitinated DGCR8 in vitro (Supplementary Fig. 4j), suggesting that DGCR8 is a substrate of USP51.

Interestingly, USP51, but not USP36, was upregulated upon Xray radiation (Fig. 5a). In both LM2 and MCF-7 cells, IR-induced upregulation of DGCR8 was reversed by shRNA-mediated knockdown of USP51, but not by knockdown of USP36 (Fig. 5b and Supplementary Fig. 5a). Moreover, the interaction between DGCR8 and USP51 was enhanced in a time-dependent manner after irradiation of LM2 cells (Supplementary Fig. 5b), and this increased interaction was observed with endogenous proteins (Fig. 5c). In addition, the K48 linkage-specific deubiquitination of DGCR8 by USP51 was enhanced by IR treatment (Fig. 5d). Altogether, these data suggest that radiation-induced USP51 mediates deubiquitination and stabilization of DGCR8.

Similar to the DGCR8-knockdown effects (Fig. 3a, b, f), knockdown of USP51 not only decreased HR and NHEJ repair efficiency in LM2-DRR cells (Fig. 5e and Supplementary Fig. 5c, d), but also abrogated the recruitment of RNF168, 53BP1, and BRCA1 to DSBs after radiation treatment, without affecting the recruitment of MDC1 and RNF8 (Fig. 5f and Supplementary Fig. 6). Moreover, the mono-, di-, and poly-ubiquitination of H2A induced by RNF8 and RNF168 upon IR was abolished by USP51 knockdown (Fig. 5g). Therefore, USP51 is required for the recruitment of the DGCR8-RNF168 complex to DSBs, histone ubiquitination, and DNA repair after irradiation.

To validate the association between DGCR8 and USP51 in patients with breast cancer, we immunohistochemically stained these two proteins in breast tumors $(n=139)$ from patients with a long-term ( $\sim 12$ years) follow-up record (Supplementary Fig. 7 a).
A positive correlation between USP51 and DGCR8 was observed in these breast carcinomas, in which $73 \%$ (61 of 83) of the tumors with moderate or weak DGCR8 expression exhibited moderate or weak USP51 expression, and 75\% (42 of 56) of the tumors with strong DGCR8 expression showed strong USP51 expression (Spearman correlation $R=0.48, P=3 \times 10^{-9}$; Supplementary Fig. $7 \mathrm{a}, \mathrm{b})$. We also plotted the USP51 protein score versus the DGCR8 protein score for individual patients, which revealed a highly significant correlation (Pearson $R^{2}=0.52, P<1 \times 10^{-15}$; Supplementary Fig. $7 c$ ), indicating the relevance of the identified regulatory mechanism in human cancer. Furthermore, KaplanMeier analysis showed that high levels of DGCR8 (log-rank $P=2 \times 10^{-10}$ ) and USP51 (log-rank $P=0.0001$ ) were significantly associated with poor overall survival after surgery in patients with breast cancer (Supplementary Fig. 7d, e).

ATM-mediated phosphorylation of DGCR8 promotes its upregulation and function upon irradiation. We investigated whether and how DGCR8 protein is regulated by upstream signaling in DDR. By using a phospho-S/TQ (p-S/TQ) antibody, we found that either WT DGCR8 or $\triangle 692$-DGCR8 was phosphorylated at ATM/ATR consensus motifs in LM2 and HEK293T cells upon IR treatment (Fig. 6a and Supplementary Fig. 8a), and that an ATM kinase inhibitor, Ku55933, abolished radiation-induced S/TQ phosphorylation of DGCR8 (Fig. 6a and Supplementary Fig. 8a), suggesting that DGCR8 is phosphorylated in an ATM-dependent manner after radiation. We also detected the phospho-S/TQ signal of endogenous DGCR8 protein in LM2-R cells, which exhibited higher basal levels of ATM phosphorylation than parental LM2 cells, and the S/T-Q phosphorylation of endogenous DGCR8 was upregulated after IR treatment of both LM2 and LM2-R cells (Fig. 6b). Analysis of the DGCR8 protein sequence revealed one evolutionarily conserved ATM phosphorylation site, serine 677 (S677; Fig. 6c and Supplementary Fig. 8b). Importantly, mass spectrometric analysis provided direct evidence that radiation treatment induced the phosphorylation of DGCR8 at S677 (Fig. 6d, e). Consistently, substitution of $\mathrm{S} 677$ with alanine or aspartic acid (S677A or S677D) abrogated the S/T-Q phosphorylation of either WT DGCR8 or $\triangle 692$-DGCR8 in irradiated HEK293T cells, further validating that $\mathrm{S} 677$ is indeed the site of radiation-induced ATMdependent phosphorylation (Fig. 6f). To determine whether DGCR8 is a substrate of ATM, we performed in vitro kinase assays with purified proteins, finding that ATM phosphorylated either full-length DGCR8 protein or $\triangle 692$-DGCR8, but not their S677A mutants (Fig. 6g). In addition, radiation-induced S677 
a

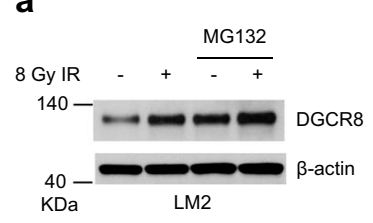

b

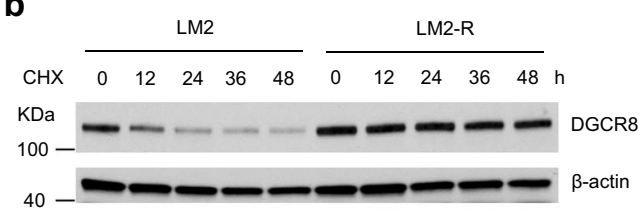

C

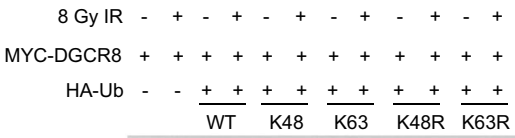

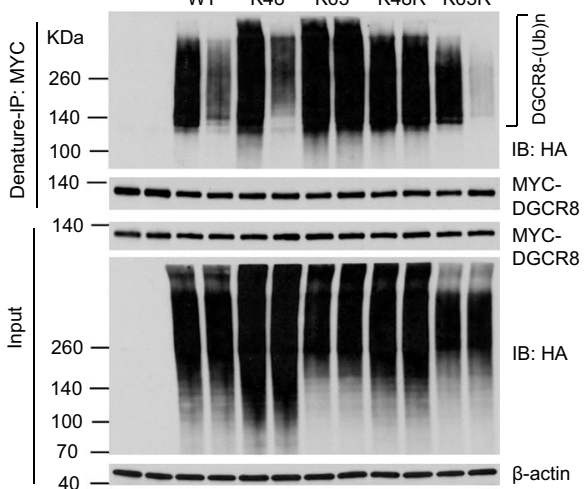

e

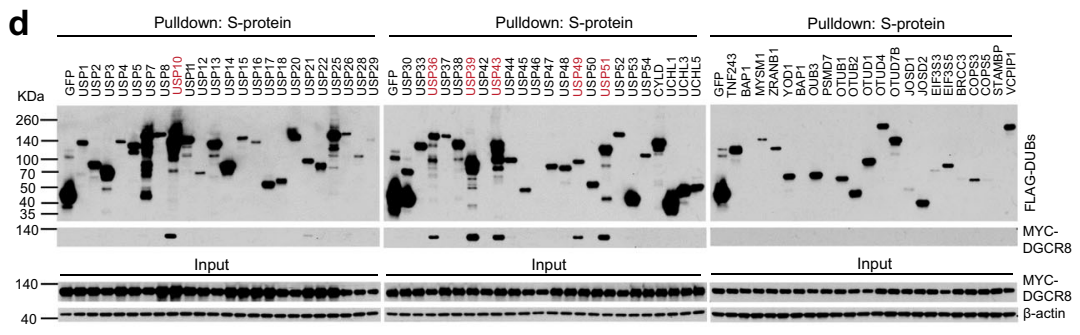

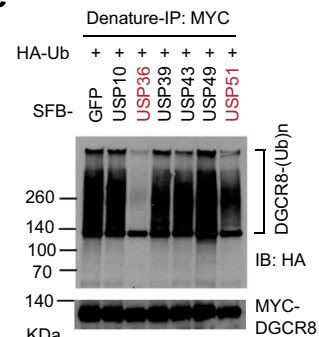
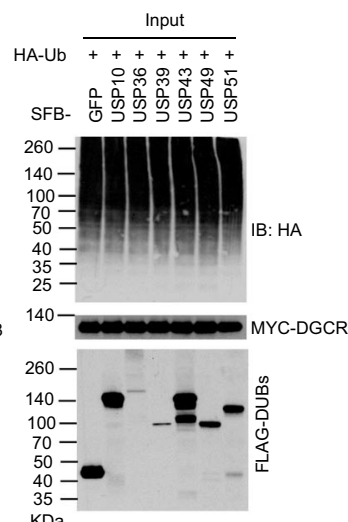

h
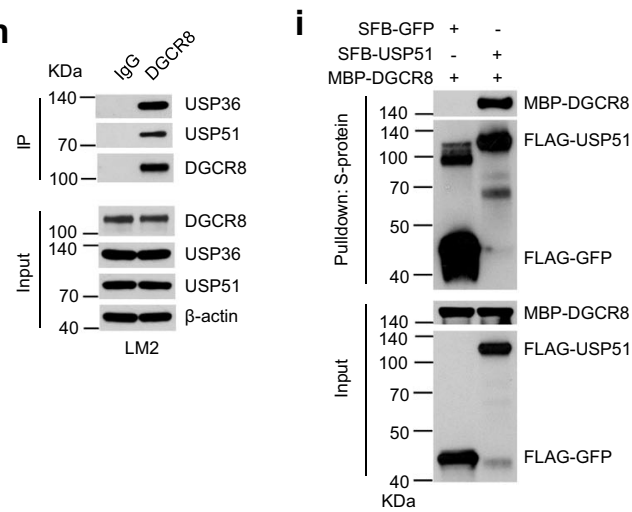

f

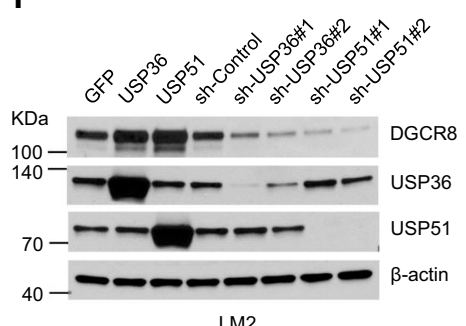

LM2 g

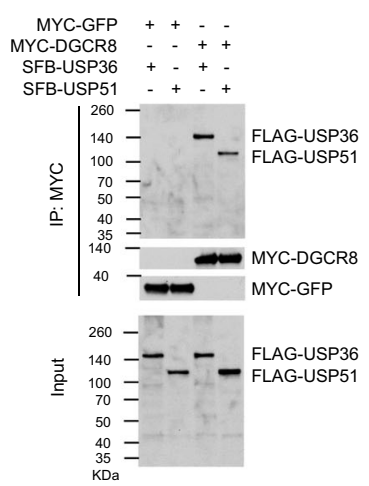

j
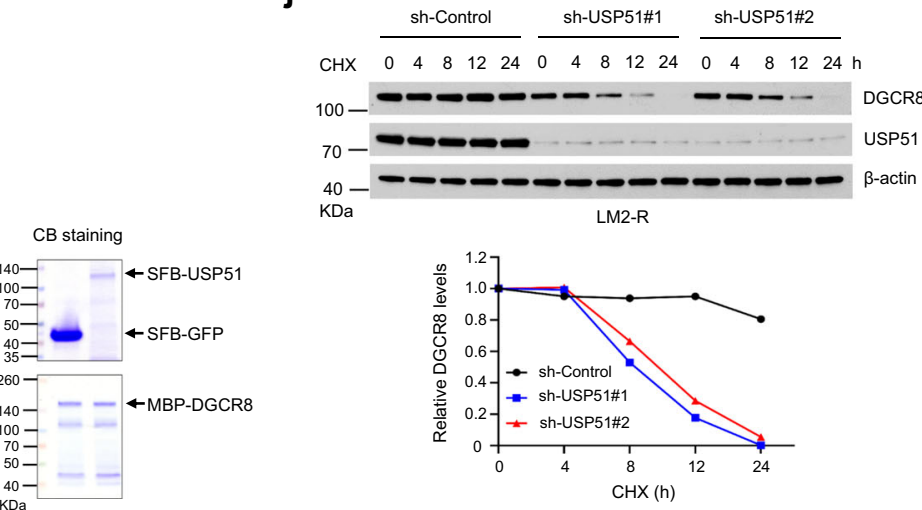

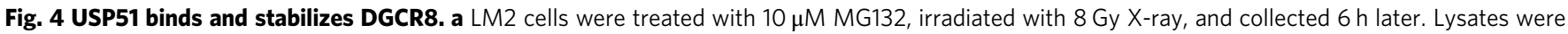
immunoblotted with antibodies against DGCR8 and $\beta$-actin. b Parental and radioresistant LM2 cells were treated with $100 \mu \mathrm{g} / \mathrm{ml} \mathrm{cycloheximide} \mathrm{(CHX).}$ Cells were collected at different time points and immunoblotted with antibodies against DGCR8 and $\beta$-actin. $\mathbf{c}$ HEK293T cells were co-transfected with MYC-DGCR8 and HA-ubiquitin ( Ub) or its lysine-specific mutants (K48, K63, K48R, or K63R), followed by immunoprecipitation with anti-MYC beads and

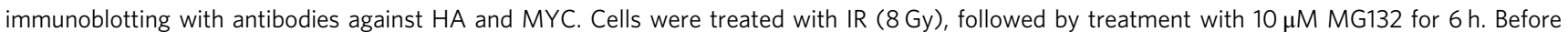
immunoprecipitation, lysates were heated at $95^{\circ} \mathrm{C}$ for $5 \mathrm{~min}$ in the presence of $1 \%$ SDS (for denaturing), followed by a 10 -fold dilution with lysis buffer and sonication. d SFB-tagged DUBs were individually co-transfected with MYC-DGCR8 into HEK293T cells, followed by pulldown with S-protein beads and immunoblotting with antibodies against FLAG and MYC. e HEK293T cells were co-transfected with MYC-DGCR8, HA-ubiquitin, and a candidate DUB. After treatment with MG132 for $6 \mathrm{~h}$, cells were lysed, denatured, and subjected to immunoprecipitation with anti-MYC beads and immunoblotting with antibodies against HA and MYC. f Immunoblotting of DGCR8, USP36, USP51, and $\beta$-actin in LM2 cells with overexpression or knockdown of USP36 or USP51. $\mathbf{g}$ HEK293T cells were co-transfected with MYC-DGCR8 and SFB-tagged USP36 or USP51. After $48 \mathrm{~h}$, cells were lysed, immunoprecipitated with anti-MYC beads, and immunoblotted with antibodies against FLAG and MYC. h Co-IP of endogenous DGCR8 with endogenous USP36 and USP51. DGCR8 was immunoprecipitated from LM2 cells and immunoblotted with antibodies against USP36, USP51, and DGCR8. i USP51 binds DGCR8 in vitro. Left panel: SFB-GFP or SFB-USP51 was retained on S-protein beads and incubated with purified MBP-DGCR8. The bound proteins were eluted by boiling in Laemmli buffer and immunoblotted with antibodies against MBP and FLAG. Right panel: purified SFB-GFP, SFB-USP51, and MBP-DGCR8 proteins were analyzed by SDS-PAGE and Coomassie blue (CB) staining. $\mathbf{j}$ Upper panel: LM2-R cells stably infected with USP51 shRNA were treated with $100 \mu \mathrm{g} / \mathrm{ml} \mathrm{CHX}$ for the indicated times. Lysates were subjected to immunoblotting with antibodies against DGCR8, USP51, and $\beta$-actin. Lower panel: DGCR8 levels were quantitated and normalized to $\beta$-actin. Source data are provided as a Source Data file. 
a

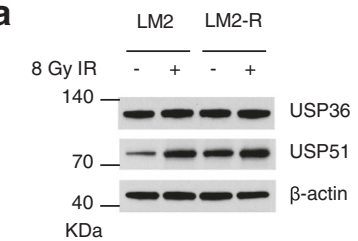

C

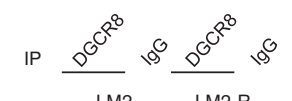

8 Gy IR $\frac{\text { LM2 }}{-++} \frac{\text { LM2-R }}{-++}$

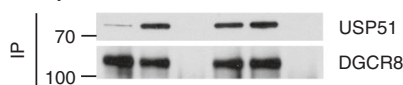

$\mid \begin{array}{ll}100- & \text { DGCR8 (SE) } \\ 100-0- & \text { DGCR8 (LE) } \\ 70-0-10 & \text { USP51 (SE) } \\ 70-10 & \text { USP51 (LE) } \\ 40- & \end{array}$

d

8 Gy IR . + + + . + + + + + + ++++

SFB-USP51 - WT Mut - WT Mut - WT Mut - WT Mut - WTMUt - WT Mut - WT Mut - WT Mut

MYC-DGCR8 +++++++++++++++++++++++

HA-Ub - . - $\frac{++++++t+++++\frac{++++++}{\mathrm{WT}}}{\mathrm{K} 48}$
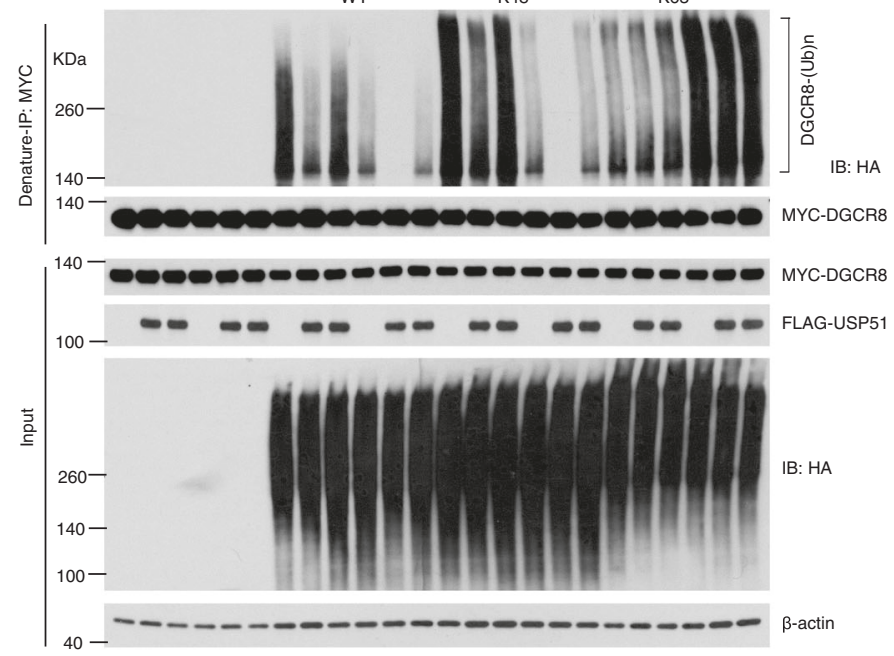

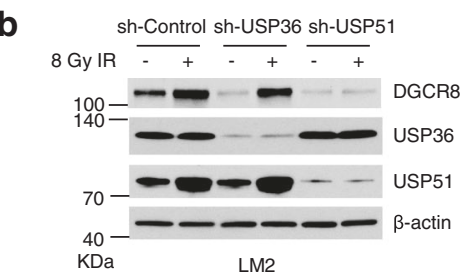

e

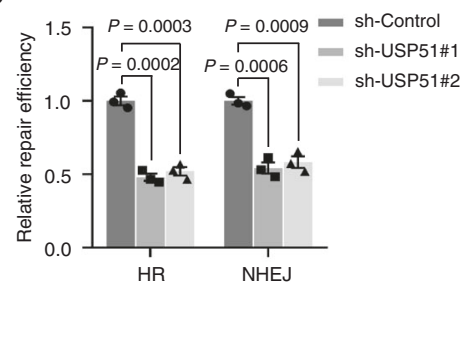

g

g sh-USP51 sh-USP51

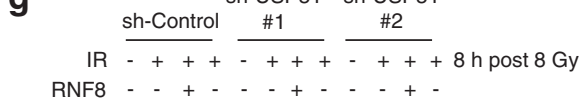

RNF168 - - + - + - - +

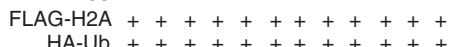

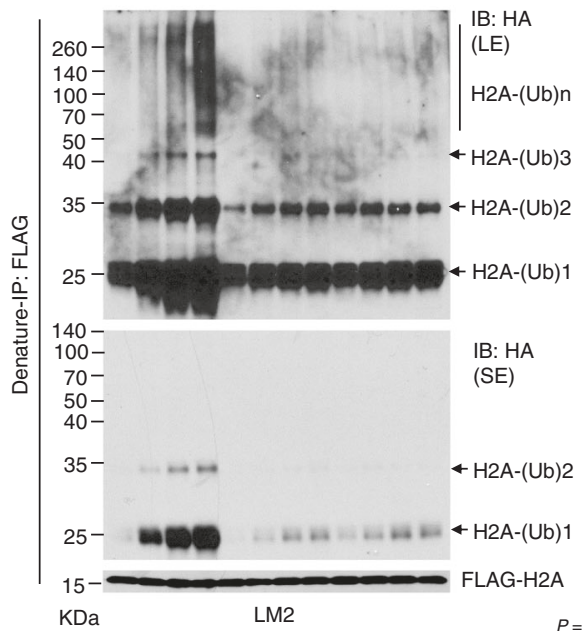

f
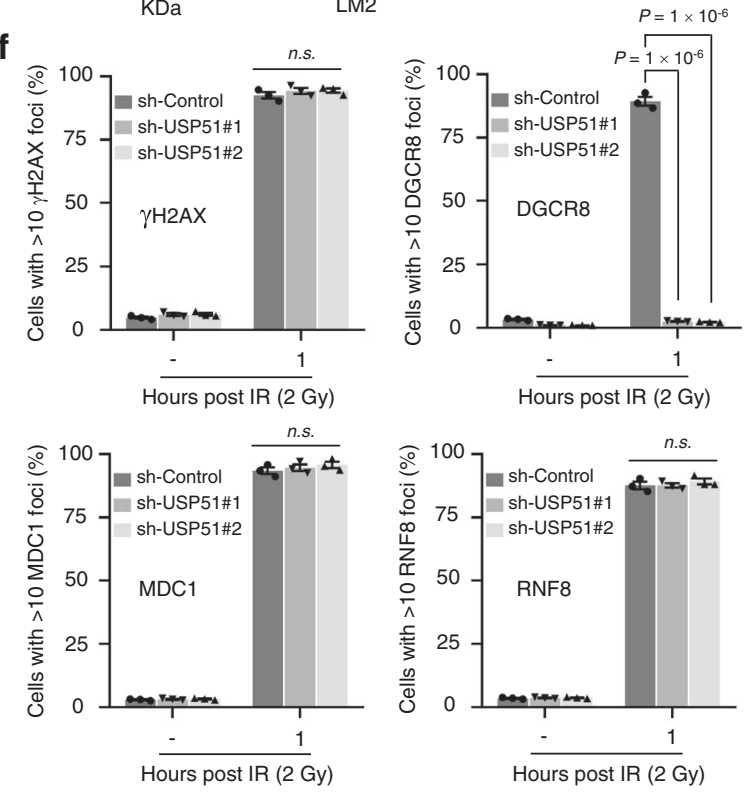
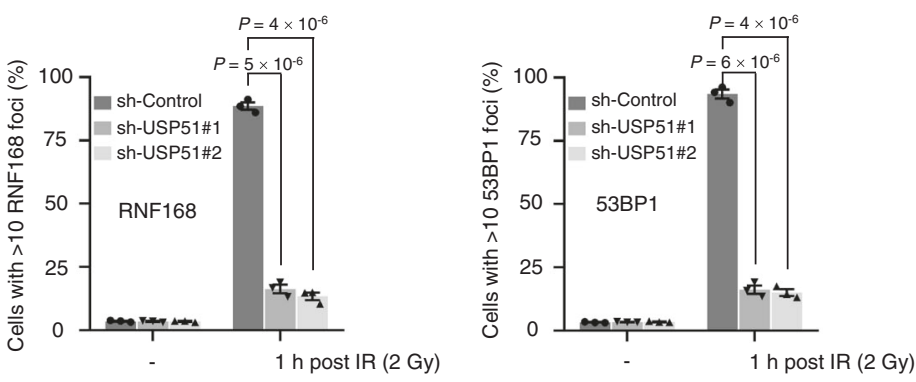

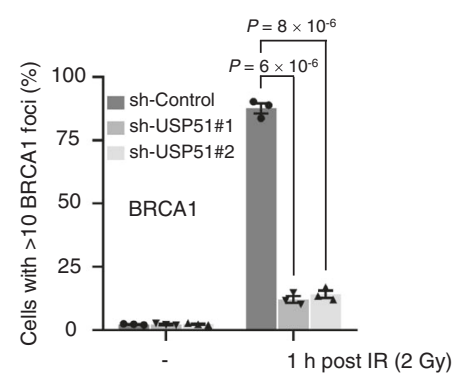

phosphorylation of DGCR8 was further validated by using an antibody against the phosphorylated S677 site of DGCR8 in both HEK293T (Fig. 6h) and LM2 (Fig. 6i) cells.

We asked whether ATM regulates DGCR8 by phosphorylating it at S677. Indeed, IR-induced upregulation of DGCR8 in LM2 cells was blocked by either the ATM inhibitor Ku55933 (Fig. 7a) or ATM shRNA (Fig. 7b), and similar effects were also observed in BT549 cells (Supplementary Fig. 8c, d). In contrast, neither treatment with the ATR inhibitor AZD6788 nor knockdown of ATR affected DGCR8 protein levels upon IR treatment
(Supplementary Fig. 8e, f). Next, we stably expressed WT DGCR8, the phosphodeficient mutant (S677A), or the phosphomimetic mutant (S677D) in DGCR8-depleted LM2 and LM2-R cells (Supplementary Fig. 8g, h). Without irradiation of LM2 cells, the stability of WT DGCR8 was higher than that of the S677A mutant but lower than that of the S677D mutant (Fig. 7c); after irradiation of LM2 cells, the stability of WT DGCR8 was increased to a level as high as that of the S677D mutant, whereas the S677A mutant was much less stable (Fig. 7c). It should be noted that the S677A mutant of DGCR8 exhibited a shorter half-life than WT DGCR8 
Fig. 5 Radiation-induced USP51 mediates deubiquitination of DGCR8, histone ubiquitination, recruitment of DDR proteins to DSBs, and DNA repair. a Immunoblotting of USP36, USP51, and $\beta$-actin in parental and radioresistant LM2 cells with and without IR treatment (8 Gy followed by $24-\mathrm{h}$ incubation). b Immunoblotting of DGCR8, USP36, USP51, and $\beta$-actin in USP36-knockdown and USP51-knockdown LM2 cells with or without IR treatment (8 Gy followed by 24-h incubation). c Co-IP of endogenous DGCR8 with endogenous USP51. LM2 and LM2-R cells were treated with 8-Gy IR. After 8 h, cells were lysed, immunoprecipitated with a DGCR8-specific antibody, and immunoblotted with antibodies against USP51 and DGCR8. SE short exposure, LE long exposure. d HEK293T cells with stable overexpression of MYC-DGCR8 were co-transfected with SFB-USP51 (wild-type or the C372S mutant) and HA-tagged ubiquitin or the lysine-specific mutant (K48 or K63), and then treated with IR (8 Gy). After 8 h, cells were lysed, denatured, and subjected to immunoprecipitation with anti-MYC beads and immunoblotting with antibodies against HA and MYC. e Knockdown of USP51 decreased HR and NHEJ efficiency in LM2-DRR cells. Two days after co-transfection of I-Scel endonuclease and an exogenous donor for HR (pCAGGS DRR mCherry Donor EF1a BFP) into the USP51-knockdown LM2-DRR cells, the percentages of GFP-positive and mCherry-positive cells, gated on BFP-positive cells, were determined by flow cytometry. Repair by HR or NHEJ leads to mCherry or GFP expression. Data were normalized to the control cells. $n=3$ biological replicates. f Quantification of $\gamma \mathrm{H} 2 \mathrm{AX}$, DGCR8, MDC1, RNF8, RNF168, 53BP1, and BRCA1 foci in USP51-knockdown LM2 cells. Cells were incubated for $1 \mathrm{~h}$ after 2-Gy IR and immunostained with antibodies against $\gamma H 2 A X, D G C R 8, M D C 1, R N F 8, R N F 168,53 B P 1$, and BRCA1 (see representative images in Supplementary Fig. 6). $n=3$ biological replicates. $g$ Control and USP51-knockdown LM2 cells with stable overexpression of FLAG-H2A and RNF8 or RNF168 were transfected with HA-ubiquitin ( $\mathrm{Ub}$ ), treated with IR ( $8 \mathrm{~Gy}$ ), and cultured for $8 \mathrm{~h}$, followed by immunoprecipitation with anti-FLAG beads and immunoblotting with antibodies against HA and FLAG. Before immunoprecipitation, lysates were heated at $95^{\circ} \mathrm{C}$ for 5 min in the presence of $1 \%$ SDS (for denaturing), followed by a 10-fold dilution with lysis buffer and sonication. Statistical significance in $\mathbf{e}$ and $\mathbf{f}$ was determined by a two-tailed unpaired $t$-test. Error bars are mean \pm SEM. Source data are provided as a Source Data file.

even without radiation treatment (Fig. 7c), which was likely due to the basal S677 phosphorylation of DGCR8 (Fig. 6h, i). In the radioresistant LM2-R cells, the stability of WT DGCR8 was much higher than that of the S677A mutant but was comparable to that of the S677D mutant (Fig. 7d). Therefore, ATM-dependent phosphorylation of DGCR8 at S677 is important for the IRinduced stabilization of DGCR8. Furthermore, we determined the HR and NHEJ repair efficiency in the DGCR8-depleted LM2-DRR reporter cell line with re-expression of WT DGCR8, the phosphodeficient mutant (S677A), or the phosphomimetic mutant (S677D), finding that the inhibited HR and NHEJ repair efficiency caused by DGCR8 knockdown was completely rescued by WT DGCR8 or the phosphomimetic mutant S677D, but not by the phosphodeficient mutant S677A (Fig. 7e and Supplementary Fig. 8i, j). In DGCR8-depleted LM2, LM2-R, and BT549 cells, the phosphodeficient mutant S677A was less able to promote radioresistance than WT DGCR8 or the phosphomimetic mutant S677D (Fig. 7f and Supplementary Fig. 8k, l), suggesting that ATM-mediated phosphorylation of DGCR8 is critical for its regulation of radiation response.

To further explore the mechanism of 5677 phosphorylation of DGCR8 in DDR, we compared the interaction of WT DGCR8, the phosphodeficient mutant (S677A), or the phosphomimetic mutant (S677D) with MDC1, RNF8, RNF168, and USP51 at 1 or $8 \mathrm{~h}$ after IR. At both time points, radiation-induced interaction of DGCR8 with MDC1 and RNF8 was abolished by the phosphodeficient mutation S677A, whereas a phosphomimetic mutant of DGCR8 (S677D) showed constitutive interaction with MDC1 and RNF8, either with or without IR treatment (Fig. 8a, b). Consistent with the results in Supplementary Fig. 5b, the interaction between WT DGCR8 and USP51 was enhanced at $8 \mathrm{~h}$, but not at $1 \mathrm{~h}$, after IR, and this increased interaction was abolished by the phosphodeficient mutation (S677A), but not by the phosphomimetic mutation (S677D) (Fig. 8a, b). Moreover, USP51 markedly reduced the ubiquitination of either WT DGCR8 or the S677D mutant, but had little effect on the ubiquitination of the S677A mutant (Fig. 8c). In addition, immunofluorescent staining showed that the S677A mutation of DGCR8 did not affect the recruitment of MDC1 or RNF8 to DSBs, but abolished the localization of DGCR8, RNF168, 53BP1, and BRCA1 to damage sites, whereas the S677D mutant of DGCR8 exhibited similar effects to WT DGCR8 in irradiated cells (Fig. 8d and Supplementary Fig. 9). Finally, we found that the enhanced mono-, di-, and polyubiquitination of H2A by RNF8 and RNF168 upon IR was blocked by the S677A mutation (Fig. 8e). Collectively, these results suggest that after irradiation, ATM-dependent phosphorylation of DGCR8 at S677 is crucial for the recruitment of the DGCR8RNF168 complex to MDC1 and RNF8 at DSBs, as well as the subsequent interaction of DGCR8 with USP51, DGCR8 deubiquitination, histone ubiquitination, and DSB repair.

\section{Discussion}

DGCR8, a component of the microprocessor complex, is essential for miRNA biogenesis and plays important roles in development, oncogenesis, the exit of mouse embryonic stem cells from pluripotency, and the maintenance of heterochromatin organization ${ }^{64-68}$. Moreover, DGCR8 has been reported to regulate nucleotide excision repair of ultraviolet-induced lesions (pyrimidine dimers and base modifications) ${ }^{46}$ as well as degradation of double-stranded, structured RNAs ${ }^{69}$. In this study, we discovered that DGCR8 acts as a regulator of DSB repair and Xray radiosensitivity independently of its Drosha-binding ability, revealing a new non-canonical role of DGCR8.

Aberrations in DSB repair underlie resistance to radiotherapy in patients with cancer. The RNF8/RNF168-mediated ubiquitination pathway governs the recruitment of DDR proteins to the chromatin surrounding DNA damage sites, which has critical roles in the repair of radiation-induced DSBs ${ }^{7}$. How RNF168 is recruited to genomic lesions remains elusive, and an $\mathrm{X}$ factor is hypothesized to be a missing link between RNF8 and RNF $168^{18}$. Recently, histone $\mathrm{H} 1^{70,71}$ and L3MBTL2 ${ }^{72}$ have been reported as signaling intermediates between RNF8 and RNF168. Here we identify DGCR8 as a previously undescribed molecular link between RNF8 and RNF168. Upon IR, phosphorylation by ATM and subsequent stabilization by USP51 promote the recruitment of DGCR8 and its constitutive binding partner RNF168 to MDC1 and RNF8 at DSBs, resulting in amplified histone ubiquitination and enhanced DSB repair (Supplementary Fig. 10). These findings suggest that radiation treatment may lead to therapy-induced radioresistance through DGCR8. It is worth mentioning that in our study, depletion of RNF168 inhibited both HR and NHEJ repair in LM2 cells; however, the effect of RNF168 on HR repair seems to be complex and tissue type-dependent, since both HRpromoting and HR-inhibiting effects were reported ${ }^{73-76}$. Thus, future studies of the role of DGCR8 in HR repair in other tissue types are warranted.

Posttranslational modifications of DGCR8, including phosphorylation and SUMOylation, have been shown to regulate DGCR8's stability and function ${ }^{77,78}$. However, the regulations 
a

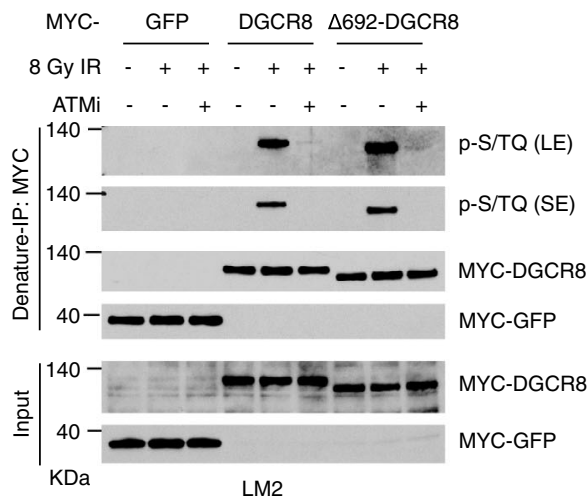

d

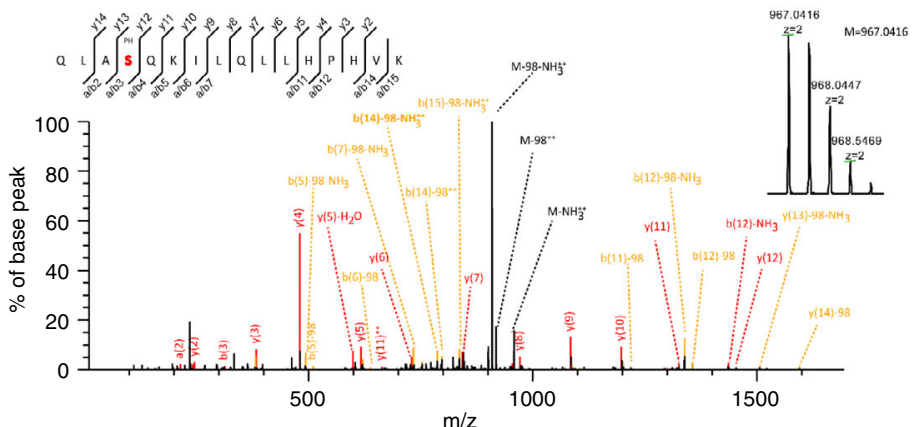

f

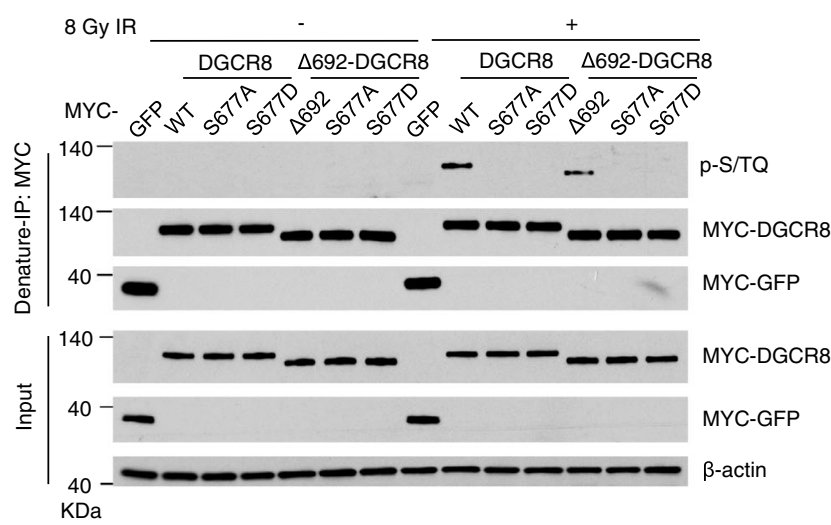

h

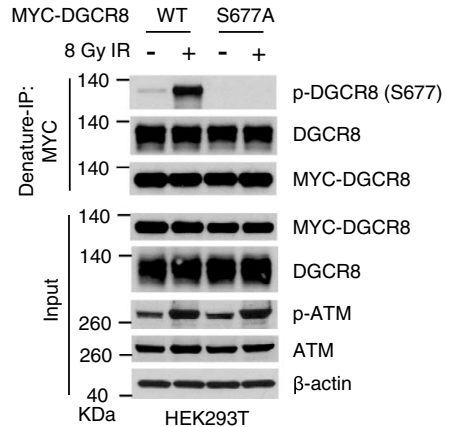

i MYC-DGCR8 WT S677A

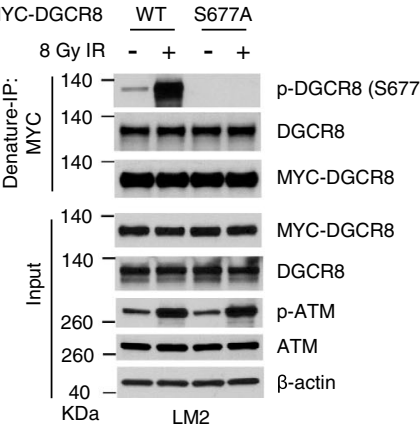

b

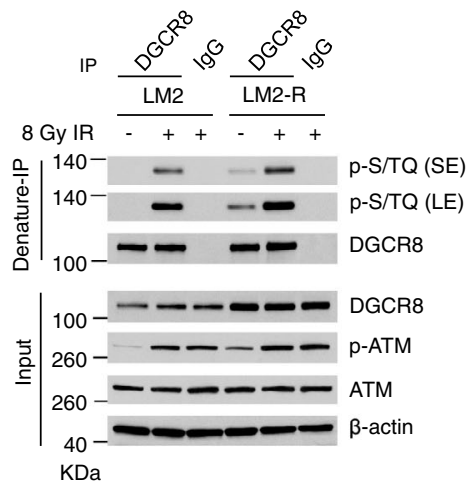

Homo sapiens

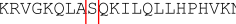
Pan troglodytes CKNKRVGKQLASSKILQLLHPHVKN Mus musculus CKNKRVGKQLASQKILQLLHPHVKN Rattus norvegicus CKNKRVGKQLA.SPKILQLLHPHVKN BOS Taurus CKNKRVGKQLASSQKILQLLHPHVKN Xenopus CKNKRVGKQLASSQKILQLLHPHVKN Ovis aries CKNKRVGKQLA.SQKILQLLHPHVRN Danio rerio CKNKRVGKQLASSQKILQMLHPHVKN

e

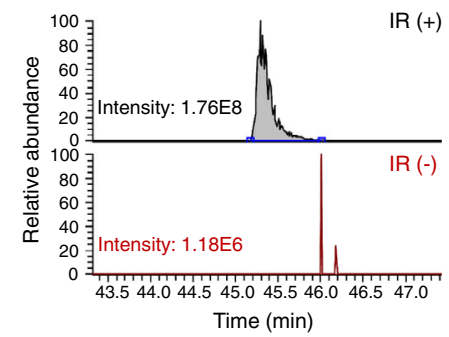

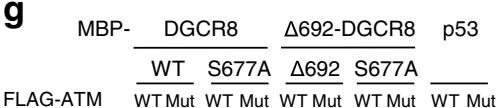

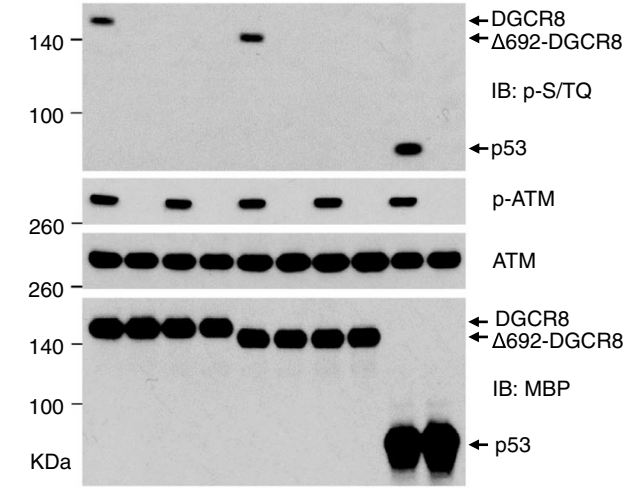

and regulators of DGCR8 ubiquitination were largely unexplored. Our study revealed the impact of phosphorylation and ubiquitination on DGCR8, and both modifications are regulated by radiation. Importantly, we found that DGCR8 is phosphorylated by ATM at serine 677, which is critical for DGCR8 stabilization and the recruitment of DGCR8 to MDC1 and RNF8 at DSBs. Moreover, by screening a DUB library, we identified USP51 and USP36 as two DUBs that interact with DGCR8 and reduce its poly-ubiquitination in cells. Although both DUBs positively regulate DGCR8 protein levels, only USP51, but not USP36, is upregulated upon radiation and is required for IR-induced expression of DGCR8. The mechanism by which USP51 is upregulated by radiation remains to be determined. Furthermore, our results demonstrate that USP51 directly binds and deubiquitinates DGCR8 in a K48 linkage-specific manner, and that this deubiquitination is enhanced by IR treatment. In addition, our results demonstrate that USP51 facilitates histone ubiquitination and DSB repair. On the other hand, Wang et al. showed that USP51 can deubiquitinate H2A at later stages of DDR in U2OS cells, and that from $4 \mathrm{~h}$ post-IR, the 53BP1 protein recruited to 


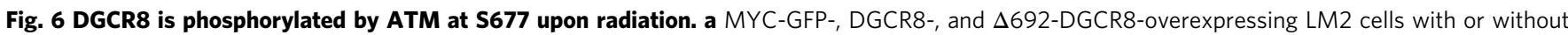
ATM inhibitor (ATMi) Ku55933 pretreatment $(10 \mu \mathrm{M}, 1 \mathrm{~h}$ ) were treated with IR ( $8 \mathrm{~Gy}$ ) and cultured for 30 min, followed by pulldown with anti-MYC beads and immunoblotting with antibodies against $\mathrm{p}-\mathrm{S} / \mathrm{TQ}$ and MYC. LE long exposure, SE short exposure. $\mathbf{b} \mathrm{LM} 2$ and LM2-R cells were treated with IR (8 Gy) and cultured for $30 \mathrm{~min}$, followed by immunoprecipitation with a DGCR8-specific antibody and immunoblotting with antibodies against $\mathrm{p}$-S/TQ and DGCR8. c Consensus ATM phosphorylation site on human DGCR8 (S677) and alignment with the conserved site on Dgcr8 from other species. d Annotated tandem mass spectrometry (MS/MS) spectrum of the peptide encompassing phosphorylated serine 677 (QLASphosQKILQLLHPHVK) of DGCR8. MYC-DGCR8-overexpressing LM2 cells with or without IR (8 Gy followed by 1-h incubation) were lysed, denatured, and subjected to immunoprecipitation with anti-MYC beads, followed by MS analysis. $\mathrm{PH}$ indicates phosphorylation. $\mathrm{NH}_{3}, \mathrm{H}_{2} \mathrm{O}$, and ++ indicate ammonia, water, and double charges, respectively. The graph shows the mass-to-charge ratios $(\mathrm{m} / \mathrm{z})$ of the doubly charged precursor peptide ions. The $x$ and $y$ axes represent $\mathrm{m} / \mathrm{z}$ and relative ion intensity, respectively. e The extracted ion chromatograms for S677phos (peptide QLAS(phos)QKILQLLHPHVK) in non-irradiated and irradiated LM2 cells based on high-performance liquid chromatography (HPLC)-MS/MS analysis. The $x$ and $y$ axes represent the retention time of HPLC/ MS analysis and the MS intensity, respectively. The area under the curve is used to indicate the relative abundance of S677phos with or without IR treatment. $\mathbf{f H E K 2 9 3 T}$ cells were transfected with DGCR8 (full-length or $\Delta 692$ ), S677A, or S677D mutant, treated with IR (8 Gy), and cultured for 30 min, followed by immunoprecipitation with anti-MYC beads and immunoblotting with antibodies against $\mathrm{p}-\mathrm{S} / \mathrm{TQ}$ and MYC. $\mathbf{g}$ In vitro kinase assay. Purified WT DGCR8 and $\triangle 692$-DGCR8 or their S677A mutants were incubated with purified wild-type ATM or the kinase-dead mutant in kinase buffer. After the reaction, proteins were resolved by SDS-PAGE and subjected to immunoblotting with the indicated antibodies. Purified MBP-p53 was used as a positive control for ATM kinase activity. h, i HEK293T (h) and LM2 (i) cells were transfected with MYC-tagged WT DGCR8 or the S677A mutant, treated with IR ( $8 \mathrm{~Gy}$ ), and cultured for $30 \mathrm{~min}$, followed by immunoprecipitation with anti-MYC beads and immunoblotting with antibodies against p-DGCR8 (S677), DGCR8, and MYC.

DSBs diminished at a slower rate in USP51-depleted U2OS cells than in control cells ${ }^{31}$. Thus, future studies are needed to dissect the different effects of USP51 on H2A ubiquitination at early and late stages of DDR. In contrast to the reduction of proteolytic, K48-linked ubiquitination of DGCR8 by radiation, IR leads to an increase in non-proteolytic, K63-linked ubiquitination of DGCR8, and this type of ubiquitination is not affected by USP51 (Fig. 5d). It is worth mentioning that our mass spectrometric analysis identified several DGCR8-interacting ubiquitin ligases, including RNF8, RNF168, CHIP, HUWE1, ZFP91, RBBP6, and ZNF598. Future studies are needed to determine which E3 ligase regulates K48-linked ubiquitination of DGCR8, whether nonproteolytic ubiquitination of DGCR8 is involved in DDR, and which E3 ligase and deubiquitinase regulate K63-linked ubiquitination of DGCR8. Interestingly, the removal of K63-linked poly-ubiquitination of certain proteins, such as TXNIP and RIP1, can trigger K48-linked ubiquitination and degradation ${ }^{79,80}$. Thus, the relationship between K63-linked and K48-linked ubiquitination of DGCR8 during DDR is worth further investigation. Finally, our work calls for the development of small-molecule inhibitors that target USP51's DUB activity or its interaction with DGCR8; such inhibitors have potential as tumor radiosensitizers.

\section{Methods}

Cell culture. The HEK293T, T47D, MCF-7, BT549, HepG2, HCT116, and HeLa cell lines were from the American Type Culture Collection (ATCC) and were cultured under conditions specified by the manufacturer. The LM2 cell line was from Xiang Zhang (Baylor College of Medicine) and the HEK293A cell line was from Junjie Chen's lab stock; both cell lines were cultured in DMEM supplemented with $10 \%$ fetal bovine serum and $1 \%$ penicillin and streptomycin. Short tandem repeat profiling (for cell line authentication) and mycoplasma tests were done by ATCC and MD Anderson's Characterized Cell Line Core Facility.

Generation of radioresistant sublines. The radioresistant sublines were generated from parental LM2 and MCF-7 cell lines as described in our previous publication $^{5}$. Briefly, after an 8-Gy dose of X-ray irradiation, surviving cells formed colonies. We pooled the colonies and repeated the dose two more times. The radiosensitivity of the cells derived from this selection, named LM2-R and MCF-7$\mathrm{R}$, was validated by clonogenic survival assays as described below. All experiments using these cells were performed at early passages.

Chemicals. The chemicals used to treat cells were MG132 (Santa Cruz Biotechnology, \#sc-201270), cycloheximide (Sigma, \#C7698), puromycin (ThermoFisher Scientific, \#A1113803), hygromycin B (ThermoFisher Scientific., \#10687010), blasticidin (ThermoFisher Scientific, \#R21001), G418 Sulfate (ThermoFisher Scientific, \#MT30234CR), Micrococcal Nuclease (MNase)
(ThermoFisher Scientific, \#88216), the ATM inhibitor Ku55933 (Sigma, \#SML1109), and the ATR inhibitor AZD-6738 (MedKoo, \#206114).

Plasmids and shRNA. The DGCR8 (\#10921), Drosha (\#10921), Dicer (\#10921), Exportin-5 (\#10921), HA-ubiquitin (WT: \#17608; K48R: \#17604; K48: \#17605; K63: \#17606), RNF8 (\#99396), FLAG-H2A (\#63560), p53 (\#81754), pLCN DSB Repair Reporter (DRR) (\#98895), and pCAGGS DRR mCherry Donor EF1a BFP (\#98896) constructs were from Addgene. The RNF168 plasmid was from DNASU (clone ID: HsCD00832967). The pCBA-I-SceI plasmid was from Dr Junjie Chen's lab stock. Full-length DGCR8 and the Drosha-binding domain deletion mutant ( $\triangle 692-D G C R 8)$, as well as FLAG-H2A, were amplified by PCR and cloned into the pDONR201 vector through the BP reaction. The S677A (serine to alanine) and S677D (serine to aspartic acid) mutants of full-length DGCR8 and $\triangle 692$-DGCR8 in the pDONR201 vector were generated by using the QuikChange ${ }^{\circledast}$ kit from Agilent Technologies and validated by sequencing. Full-length DGCR8, $\Delta 692$-DGCR8, and their S677A and S677D mutants, as well as FLAG-H2A, were subcloned into pcDNA3.1-MYC, pCDH-MYC, pLenti-CMV-puromycin, pLenti-CMV-hygromycin, pLX304, or MBP-tagged destination vectors through the LR reaction. The MBP-p53 construct was generated from pDONR-p53 (Addgene, \#81754) through the LR reaction. The K63R mutant of HA-ubiquitin and the 68 human DUB ORFs in the $\mathrm{pBabe}-\mathrm{SFB}$ vector were described in our previous publication ${ }^{60}$. FLAG-ATM and its kinase-dead mutant were described in our previous publication ${ }^{5}$. The GSTUSP51 and SFB-USP51-C372S plasmids were described in our previous publication $^{63}$. GFP and RNF168 were cloned into the GST-tagged destination vector through the LR reaction. USP36 and USP51 were subcloned from the pDONR201 vector into pLenti-CMV-puromycin and pLenti-CMV-hygromycin destination vectors through the LR reaction. The pLKO.1- DGCR8 shRNA (clone ID: NM_022720.5-2583s21c1 and NM_022720.4-575s1c1), USP51 shRNA (clone ID: NM_201286.1-669s1c1 and NM_201286.1-1915s1c1), USP36 shRNA (clone ID: NM_025090.3-4844s21c1 and NM_025090.3-1148s21c1), and RNF168 shRNA (clone ID: NM_152617.2-757slc1 and NM_152617.2-2122s1c1) constructs were from Sigma. The pGIPZ- ATM shRNA (clone ID: V3LHS_350469 and V3LHS_350471) and ATR shRNA (clone ID: V2LHS_94659 and V2LHS_94661) constructs were from Dharmacon. RNAi-resistant DGCR8, 4692 -DGCR8, S677ADGCR8, and S677D-DGCR8 constructs were generated by using the QuikChange kit from Agilent Technologies and were then subcloned into the pLenti-CMVhygromycin destination vector through the LR reaction.

Lentiviral transduction. Virus-containing supernatant was collected $48 \mathrm{~h}$ after cotransfection of the viral vector and packaging plasmids (psPAX2 and pMD2.G) into HEK293T cells, and was then added to the target cells. The infected cells were selected with puromycin, hygromycin B, blasticidin, or G418 for 3-14 days after infection.

Immunoblotting. Western blot analysis was performed with precast gradient gels (Bio-Rad) using standard methods. Briefly, cultured cells were lysed in RIPA buffer (Millipore, \#20-188) containing protease inhibitors (GenDEPOT, \#P3100) and phosphatase inhibitors (GenDEPOT, \#P3200). Proteins were separated by SDSPAGE and blotted onto a nitrocellulose membrane (Bio-Rad). Membranes were blocked and then probed with the specific primary antibodies, followed by peroxidase-conjugated secondary antibodies. The bands were visualized by chemiluminescence (Denville Scientific). The following antibodies were used: antibodies against DGCR8 (1:2000, Abcam, \#ab191875), Dicer (1:1000, Cell Signaling 


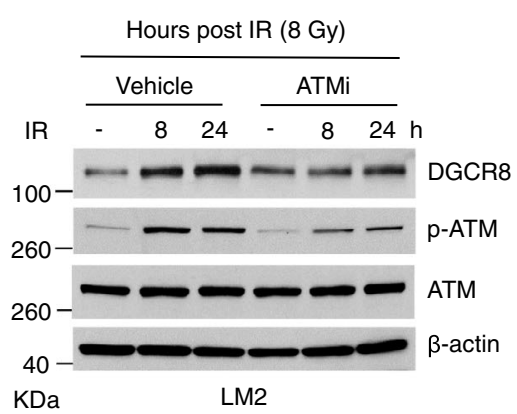

b

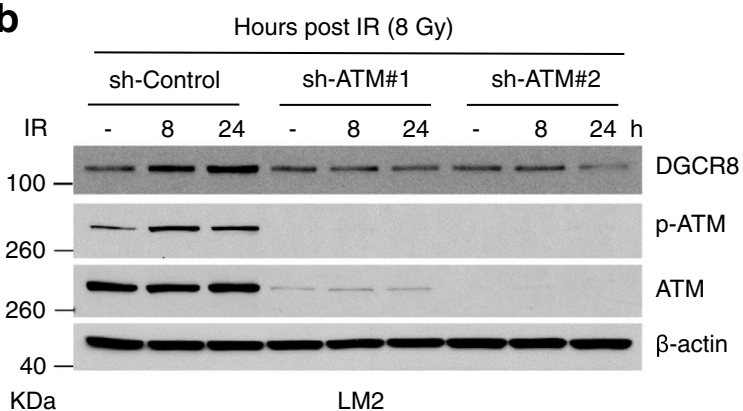

d

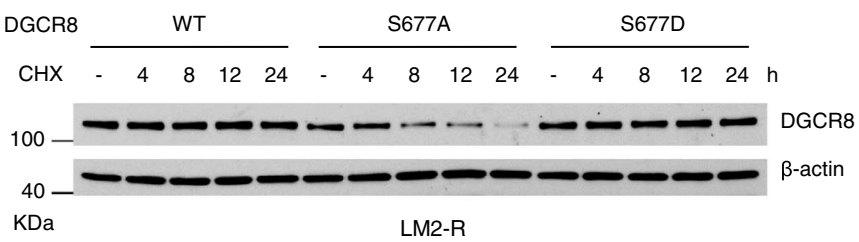

e

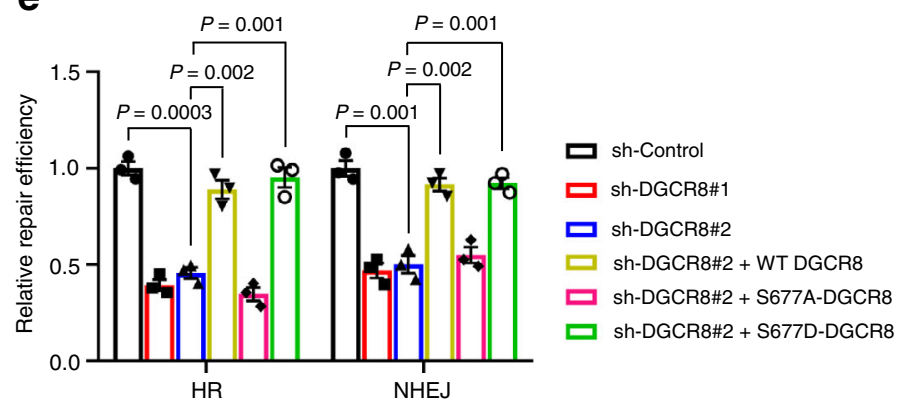

C
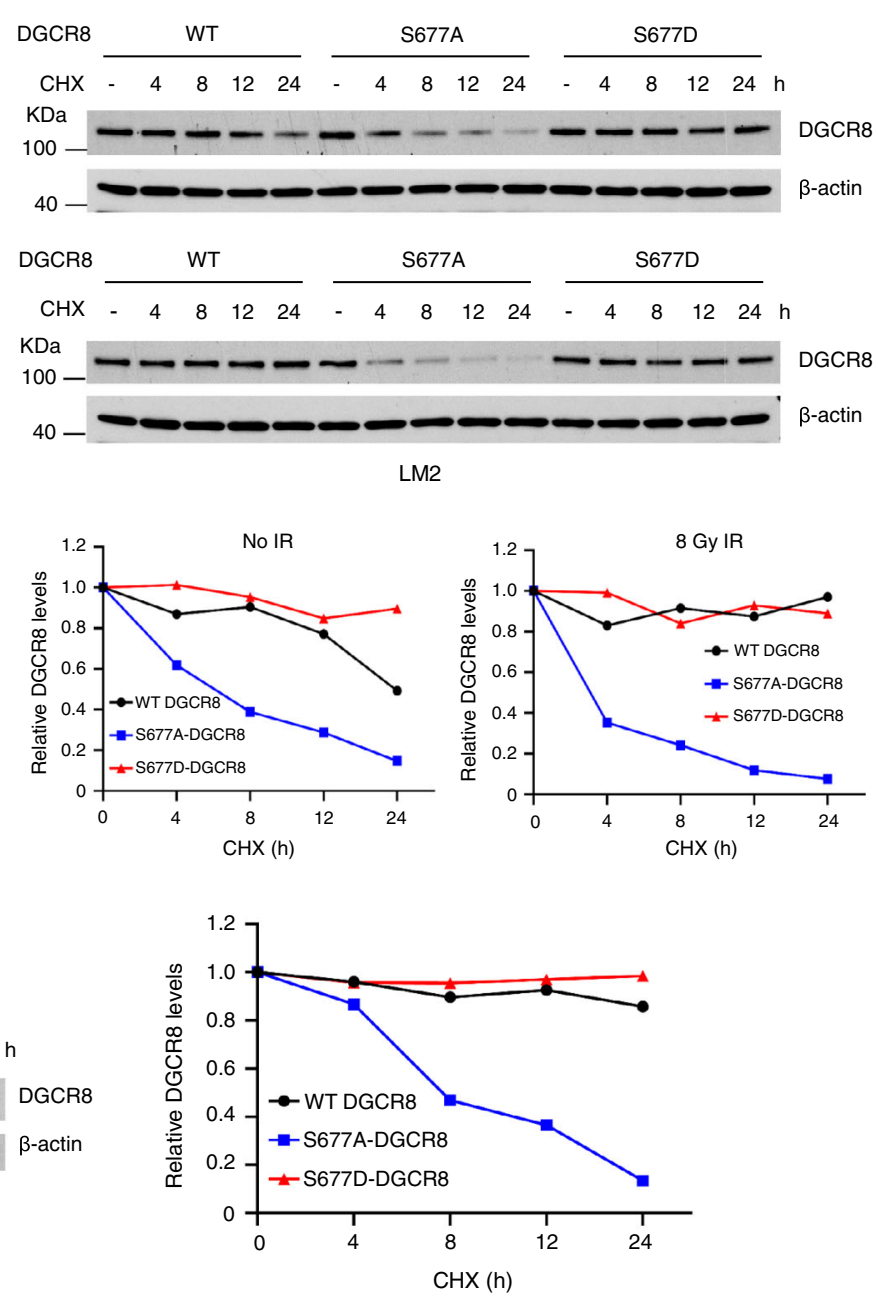

f

LM2-R

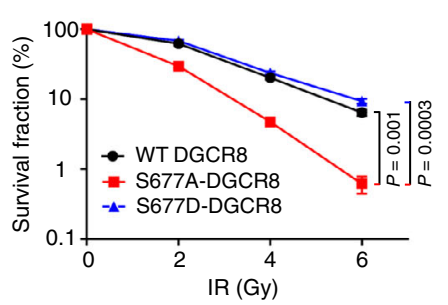

Fig. 7 ATM-mediated $\mathbf{5 6 7 7}$ phosphorylation of DGCR8 is crucial for DGCR8 stability, DSB repair, and radiation response. a LM2 cells were pretreated with ATMi (Ku55933, $10 \mu \mathrm{M}$ for $1 \mathrm{~h}$ ), followed by IR treatment ( $8 \mathrm{~Gy}$ ). Lysates were collected at the indicated times and immunoblotted with antibodies against DGCR8, p-ATM, ATM, and $\beta$-actin. b Immunoblotting of DGCR8, p-ATM, ATM, and $\beta$-actin in control and ATM-knockdown LM2 cells collected at the indicated times after IR. c Upper panels: DRCR8-knockdown LM2 cells with ectopic expression of WT DGCR8, the S677A mutant, or the S677D mutant were treated with $\mathrm{CHX}$ with or without IR, collected at different time points, and immunoblotted with antibodies against DGCR8 and $\beta$-actin. Lower panels: DGCR8 protein levels were quantitated and normalized to $\beta$-actin. $\mathbf{d}$ Left panel: DRCR8-knockdown LM2-R cells with ectopic expression of WT DGCR8, the S677A mutant, or the S677D mutant were treated with CHX, collected at different time points, and immunoblotted with antibodies against DGCR8 and $\beta$-actin. Right panel: DGCR8 protein levels were quantitated and normalized to $\beta$-actin. e The effect of S677 DGCR8 phosphorylation on HR and NHEJ efficiency in LM2-DRR cells. Two days after co-transfection of I-Scel endonuclease and an exogenous donor for HR ( $p C A G G S$ DRR mCherry Donor EF1a BFP) into DGCR8-knockdown and DGCR8-, S677A-DGCR8-, and S677D-DGCR8-rescued LM2-DRR cells, the percentages of GFP-positive and mCherrypositive cells, gated on BFP-positive cells, were determined by flow cytometry. Repair by HR or NHEJ leads to mCherry or GFP expression. Data were normalized to the control cells. $n=3$ biological replicates. $\mathbf{f}$ Clonogenic survival assays of DRCR8-knockdown LM2-R cells with ectopic expression of WT DGCR8, the S677A mutant, or the S677D mutant after X-ray IR treatment. $n=3$ wells per group. Statistical significance in $\mathbf{e}$ and $\mathbf{f}$ was determined by a two-tailed unpaired $t$-test. Error bars are mean \pm SEM. Source data are provided as a Source Data file. 
a

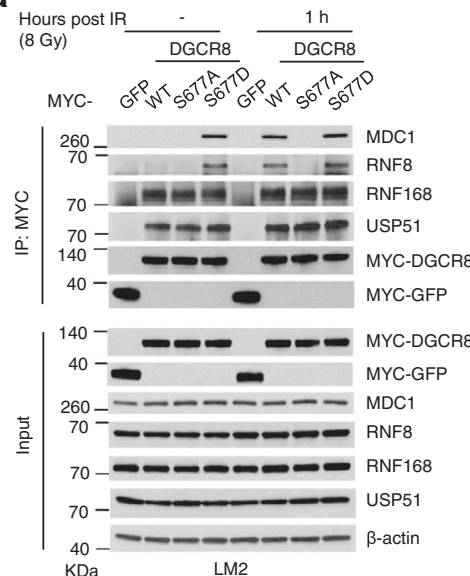

b

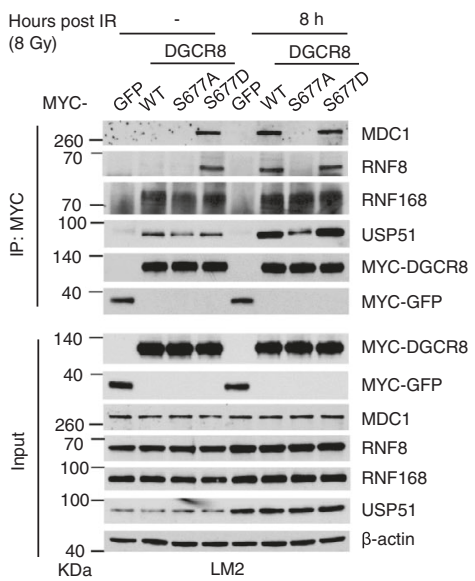

e

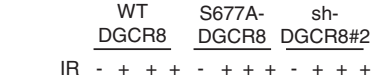

IR - +++ - +++ -+++ 8 h post 8 Gy

RNF8 - + - - + - +

FLAG-H2A ++++++++++++

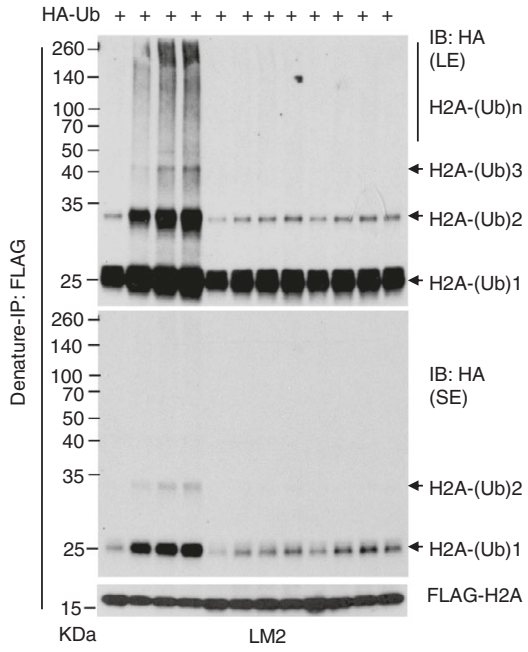

C

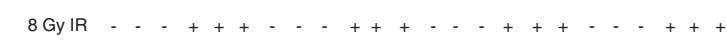

SFB-USP51 - WT Mut - WTMut - WT Mut - WT Mut - WT Mut - WT Mut - WTMut - WTMUt

HA-Ub ++++++++++++++++++++++++

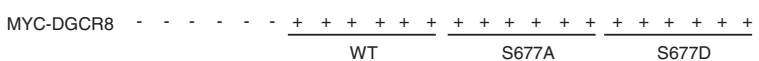
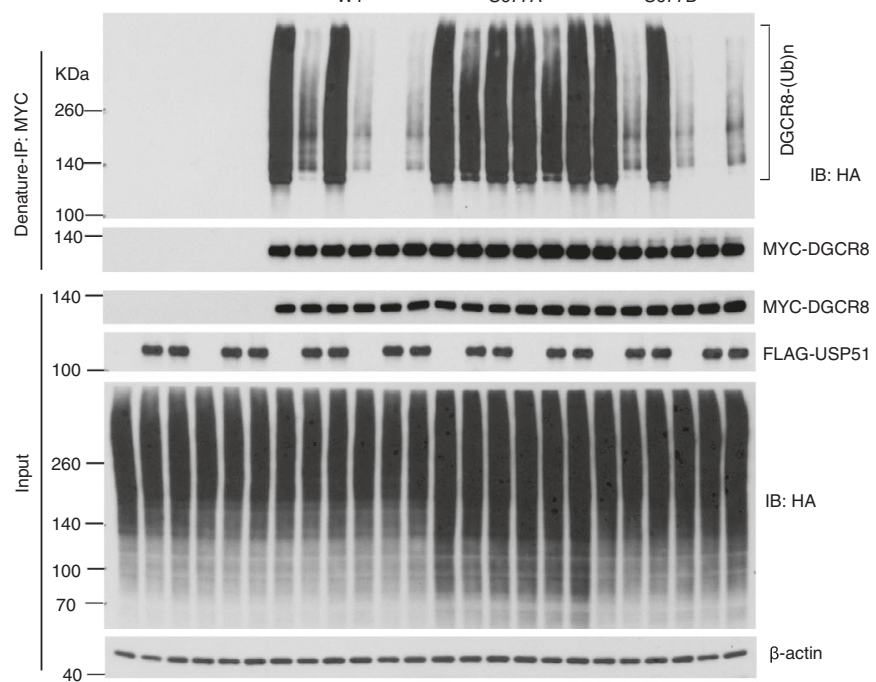

d
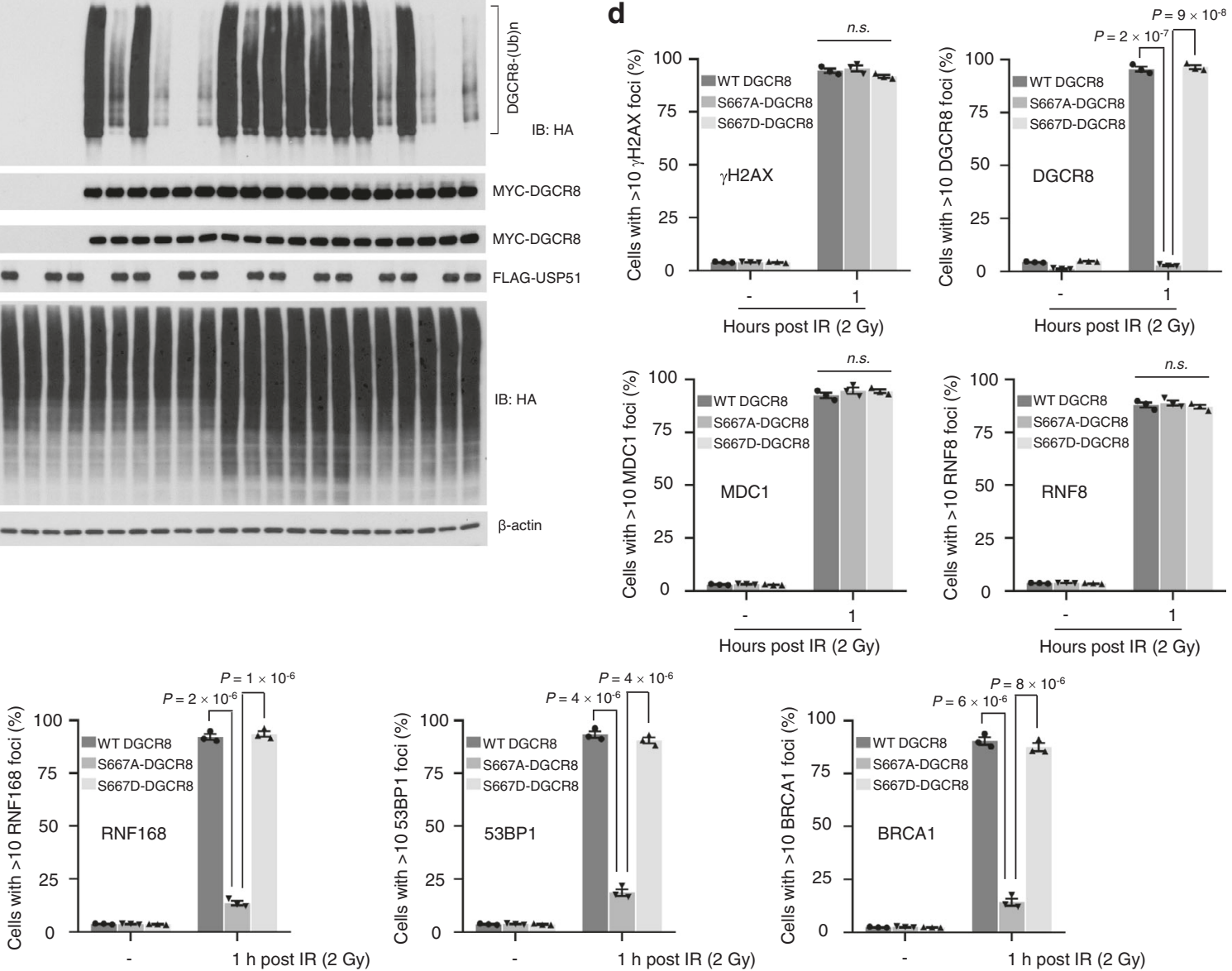

Technology, \#5362S), Drosha (1:1000, Cell Signaling Technology, \#3364S), Exportin-5 (1:1000, Cell Signaling Technology, \#12565), $\gamma \mathrm{H} 2 \mathrm{AX}$ (1:1000, Cell Signaling Technology, \#9718S), H2AX (1:1000, Cell Signaling Technology, \#2595S), H2A (1:1000, Cell Signaling Technology, \#2578S), p-CHK1 (1:1000, Cell Signaling Technology, \#12302S), CHK1 (1:1000, Cell Signaling Technology, \#2360S), p-CHK2 (1:1000, Cell Signaling Technology, \#2661S), CHK2 (1:1000, Cell Signaling Technology, \#6334S), p-ATM (1:1000, Cell Signaling Technology, \#5883S), ATM (1:1000, Cell Signaling Technology, \#2873S), p-ATR (1:1000, Cell Signaling Technology, \#2853S), ATR (1:1000, Cell Signaling Technology, \#2790S), p-S/TQ (1:1000, Cell Signaling Technology, 2851S), MBP (1:1000, Cell Signaling Technology, 2396S), GST (1:1000, Cell Signaling Technology, \#2622S), MDC1
(1:1000, R\&D Systems, \#MAB6497), RNF8 (1:1000, Millipore, \#09-813), RNF168 (1:1000, Millipore, \#ABE367), USP36 (1:1000, a gift from Dr Masayuki Komada at Tokyo Institute of Technology), USP51 (1:3000, a gift from Dr Sharon Dent at MD Anderson Cancer Center), $\beta$-actin (1:1000, Santa Cruz Biotechnology, \#sc-47778), FLAG (1:5000, Sigma, \#F3165, clone M2), HA (1:2000, Santa Cruz Biotechnology, \#sc-7392), and MYC (1:2000, Santa Cruz Biotechnology, \#sc-40, clone 9E10). The antibody against phospho-DGCR8 (pS677; 1:500) was generated at Biomatik. The ImageJ program (version 1.53g) was used for densitometric analysis of western blots, and the quantification results were normalized to an internal control. 
Fig. 8 S677 DGCR8 phosphorylation is critical for the recruitment of RNF168 to RNF8 and MDC1, DGCR8-USP51 interaction, DGCR8 deubiquitination, and histone ubiquitination upon irradiation. $\mathbf{a}$, b MYC-GFP-, WT DGCR8-, S677A-DGCR8-, and S677D-DGCR8-overexpressing LM2 cells with or without IR treatment ( $\mathbf{a}, 8 \mathrm{~Gy}$ followed by 1-h incubation; $\mathbf{b}, 8 \mathrm{~Gy}$ followed by 8-h incubation) were subjected to pulldown with MYC beads and immunoblotting with the indicated antibodies. c HEK293T cells with stable overexpression of MYC-tagged WT DGCR8, S677A-DGCR8, or S677D-DGCR8 were cotransfected with SFB-USP51 (WT or the C372S mutant) and HA-tagged ubiquitin, and then treated with IR ( 8 Gy). After 8 h, cells were lysed, denatured, and subjected to immunoprecipitation with anti-MYC beads and immunoblotting with antibodies against HA and MYC. d Quantification of $\gamma \mathrm{H} 2 \mathrm{AX}$, DGCR8, MDC1, RNF8, RNF168, 53BP1, and BRCA1 foci in DRCR8-knockdown LM2 cells with ectopic expression of WT DGCR8, S677A-DGCR8, or S677DDGCR8. Cells were incubated for $1 \mathrm{~h}$ after 2-Gy IR and immunostained with antibodies against $\gamma \mathrm{H} 2 \mathrm{AX}, \mathrm{DGCR8}, \mathrm{MDC1}, \mathrm{RNF8}, \mathrm{RNF168,53BP1}$, and BRCA1 (see representative images in Supplementary Fig. 9). $n=3$ biological replicates. Statistical significance was determined by a two-tailed unpaired $t$-test. Error bars are mean \pm SEM. e DRCR8-knockdown LM2 cells with ectopic expression of WT DGCR8 or the S677A mutant were transduced with FLAG-H2A and RNF8 or RNF168. The cells were then transfected with HA-ubiquitin (Ub), treated with IR ( $8 \mathrm{~Gy}$ ), and cultured for $8 \mathrm{~h}$, followed by immunoprecipitation with anti-FLAG beads and immunoblotting with antibodies against HA and FLAG. Before immunoprecipitation, lysates were heated at $95{ }^{\circ} \mathrm{C}$ for 5 min in the presence of $1 \%$ SDS (for denaturing), followed by a 10-fold dilution with lysis buffer and sonication. LE long exposure, SE short exposure. Source data are provided as a Source Data file.

Immunoprecipitation and pulldown assays. Cells were lysed in NETN buffer (20 mM Tris- $\mathrm{HCl}, \mathrm{pH} 8.0,100 \mathrm{mM} \mathrm{NaCl}, 1 \mathrm{mM}$ EDTA, $0.5 \%$ Nonidet P-40) or CHAPS buffer (25 mM Tris-HCl, pH 7.5, $120 \mathrm{mM} \mathrm{NaCl,} 1 \mathrm{mM}$ EDTA, $0.33 \%$ CHAPS) containing protease inhibitors and then sonicated. The chromatin fraction was isolated using a Chromatin Extraction Kit (Abcam, \#ab117152), according to the manufacturer's protocol, followed by sonication and with or without MNase treatment. For immunoprecipitation of endogenous proteins, cell extracts (lysed in CHAPS buffer) were pre-cleared with protein-A/G PLUS beads (Santa Cruz Biotechnology, \#sc-2003) and IgG at $4{ }^{\circ} \mathrm{C}$ for $30 \mathrm{~min}$, followed by incubation with an antibody against RNF8 (Proteintech, \#14112-1-AP), RNF168 (Proteintech, \#213931-AP), or DGCR8 (Bethyl Laboratories, \#A302-468A) or IgG at $4{ }^{\circ} \mathrm{C}$ for $1 \mathrm{~h}$, and were then incubated with protein-A/G PLUS beads at $4{ }^{\circ} \mathrm{C}$ overnight. The beads were washed with Tris-buffered saline containing $0.05 \%$ Tween-20 and CHAPS buffer. The bound proteins were eluted by incubation with $2 \times$ Laemmli buffer at room temperature for $10 \mathrm{~min}$ with mixing. For immunoprecipitation of tagged proteins, cell extracts were pre-cleared with protein-A/G PLUS beads and incubated with anti-MYC agarose beads (Millipore, \#A7470) at $4^{\circ} \mathrm{C}$ for $2 \mathrm{~h}$. For pulldown of SFB-tagged proteins, cell extracts were incubated with S-protein beads (Millipore, \#69704) at $4^{\circ} \mathrm{C}$ for $2 \mathrm{~h}$.

In vitro binding assay. For DGCR8-RNF168 binding, bacterially purified MBPDGCR8 was eluted with maltose and then incubated with glutathione beads (GE Healthcare, 17-0756-01) conjugated with bacterially purified GST-GFP or GSTRNF168 at $4{ }^{\circ} \mathrm{C}$ overnight. For DGCR8-USP51 binding, bacterially purified MBPDGCR8 was incubated with mammalian purified SFB-USP51 or SFB-GFP from transfected HEK293T cells, followed by pulldown with S-protein beads at $4{ }^{\circ} \mathrm{C}$ overnight. The beads were washed with NETN buffer four times and the bound proteins were eluted by boiling in $2 \times$ Laemmli buffer and subjected to western blot analysis.

Immunofluorescence. For immunostaining of $\gamma \mathrm{H} 2 \mathrm{AX}$, DGCR8, MDC1, RNF8, RNF168, BRCA1, and 53BP1, cells were cultured in chamber slides (ThermoFisher Scientific) overnight, followed by X-ray irradiation. For immunostaining of DGCR8, USP36, and USP51, HEK293A cells were co-transfected with MYCDGCR8 and SFB-tagged USP36 or USP51 and were cultured in chamber slides (ThermoFisher Scientific) overnight. The cells were washed with phosphatebuffered saline (PBS), fixed with $4 \%$ paraformaldehyde, permeabilized with $0.1 \%$ Triton X-100 in PBS, blocked with 3\% bovine serum albumin in PBS, and incubated with antibodies against $\gamma \mathrm{H} 2 \mathrm{AX}$ (1:100, Cell Signaling Technology, \#9718S), $\gamma \mathrm{H} 2 \mathrm{AX}$ (1:100, BD Biosciences, \#560443), DGCR8 (1:100, Abcam, \#ab191875), MDC1 (1:200, Bio-Rad, \#AHP799), RNF8 (1:200, Proteintech, \#14112-1-AP), RNF168 (1:100, Millipore, \#ABE367), BRCA1 (1:20, Santa Cruz Biotechnology, \#sc-6954), 53BP1 (1:100, Novus Biologicals, \#NB100-304), FLAG (1:500, Sigma, \#F7425), and MYC (1:500, Santa Cruz Biotechnology, \#sc-40, clone 9E10) at $4{ }^{\circ} \mathrm{C}$ overnight, followed by incubation with Alexa Fluor 488 goat anti-rabbit IgG (1:1000, Invitrogen, ThermoFisher Scientific, \#A-11008), Alexa Fluor 647 donkey anti-sheep IgG (1:1000, Invitrogen, ThermoFisher Scientific, \#A-21448), Alexa Fluor 488 goat anti-mouse IgG (1:1000, Invitrogen, ThermoFisher Scientific, \#A11001), Alexa Fluor 594 goat anti-rabbit IgG (1:1000, Invitrogen, ThermoFisher Scientific, \#A-11012), and/or Alexa Fluor 594 goat anti-mouse IgG (1:1000, Invi trogen, ThermoFisher Scientific, \#A-11005) at room temperature for $1 \mathrm{~h}$. Coverslips were mounted on slides by using anti-fade mounting medium with $4^{\prime}, 6$ diamidino-2-phenylindole (DAPI, Vector Laboratories, \#H-1200). Immunofluorescence images were acquired on a Zeiss LSM880 confocal microscope or Olympus FV1000 confocal microscope. Zen 2.6 (Zeiss) software was used for confocal image processing.

RNA isolation and qPCR. Total RNA was isolated using TRIzol reagent (Invitrogen) and was then reverse transcribed with an iScript complementary DNA
(cDNA) Synthesis Kit (Bio-Rad, \#1708891). The resulting cDNA was used for realtime PCR with the iTaq Universal SYBR Green Supermix (Bio-Rad, \#1725124). GAPDH was used as an internal control. Real-time PCR and data collection were performed on a CFX96 instrument (Bio-Rad). The primer sequences are listed in Supplementary Table 1.

Mass spectrometry. To identify DGCR8-interacting proteins in LM2 cells with or without IR treatment, we performed the tandem-affinity purification and mass spectrometry as follows. LM2 cells were transfected with SFB-tagged DGCR8. Forty-eight hours after transfection, the cells were cultured for $1 \mathrm{~h}$ after release from $8 \mathrm{~Gy}$ X-rays. Expression of the transfected protein was confirmed by immunoblotting. For affinity purification, a total of ten $15-\mathrm{cm}$ dishes of LM2 cells expressing SFB-DGCR8 were lysed in NETN buffer $(200 \mathrm{mM}$ Tris- $\mathrm{HCl}, \mathrm{pH} 8.0$, $100 \mathrm{mM} \mathrm{NaCl}, 0.05 \%$ Nonidet P-40, $1 \mathrm{mM}$ EDTA) containing protease inhibitors at $4{ }^{\circ} \mathrm{C}$ for $20 \mathrm{~min}$ and then sonicated. Crude lysates were cleared by centrifugation, and the supernatants were incubated with $300 \mu \mathrm{l}$ streptavidin-sepharose beads (GE Healthcare, \#17511301) at $4^{\circ} \mathrm{C}$ for $2 \mathrm{~h}$. The beads were washed three times with NETN buffer, and the bound proteins were eluted with NETN buffer containing $2 \mathrm{mg} / \mathrm{ml}$ biotin (Sigma, \#B4501) at $4^{\circ} \mathrm{C}$ for $2 \mathrm{~h}$. The eluates were incubated with $100 \mu \mathrm{l} \mathrm{S}$-protein agarose beads (Millipore, \#69704) at $4^{\circ} \mathrm{C}$ for $2 \mathrm{~h}$, and the beads were washed three times with NETN buffer. The bound proteins were eluted by boiling in $2 \times$ Laemmli buffer, resolved by SDS-PAGE, visualized by Coomassie blue staining, and subjected to in-gel digestion ${ }^{81}$.

For identifying the phosphorylation site(s) on DGCR8 upon IR, MYC-tagged DGCR8 protein immunoprecipitated from non-irradiated or irradiated LM2 cells was resolved by SDS-PAGE and visualized by Coomassie Brilliant Blue staining. DGCR8 bands were excised, destained with 50\% ethanol, and dehydrated with acetonitrile. After reduction and alkylation with dithiothreitol and iodoacetamide, bands were then subjected to in-gel digestion with trypsin (enzyme:protein $=1: 50$ ) at $37^{\circ} \mathrm{C}$ for $8 \mathrm{~h}$. The tryptic peptides were extracted with water and acetonitrile from the gel after digestion. The extracted peptides were dried by Speed-Vac and then subjected to high-performance liquid chromatography-tandem mass spectrometry analysis as follows. Tryptic peptides from in-gel digestion were dissolved in HPLC solvent A ( $0.1 \%$ formic acid in $2 \%$ acetonitrile and $\left.98 \% \mathrm{H}_{2} \mathrm{O}\right)$, injected onto a manually packed reversed-phase $\mathrm{C} 18$ column $(170 \mathrm{~mm} \times 79 \mu \mathrm{m}$, 3- $\mu \mathrm{m}$ particle size, Dikma, China) coupled to an Easy-nLC 1200 chromatography system (ThermoFisher Scientific). Peptides were eluted using a 1-h gradient of $8-80 \%$ solvent B $\left(0.1 \%\right.$ formic acid in $90 \%$ acetonitrile and $\left.10 \% \mathrm{H}_{2} \mathrm{O}\right)$ in solvent $\mathrm{A}$ at a flow rate of $300 \mathrm{nl} / \mathrm{min}$. Peptides eluted from HPLC were ionized with a nanospray source and analyzed in an Orbitrap HF-X mass spectrometer (ThermoFisher Scientific).

For the identification of the DGCR8 phosphorylation site, acquired MS raw files were transformed into MGF format using the Proteome Discoverer software (version 2.2, ThermoFisher Scientific). Proteins were identified by comparing the fragment spectra against those in the UniProt database using Mascot v.2.6.01 (Matrix Science Ltd., London, UK). For the identification of DGCR8-interacting proteins, acquired MS raw files were searched against the human proteome database from UniProt (January 26, 2019, updated, 93,798 sequences) using MaxQuant software (version 1.6.7.0), with a reversed decoy database by Andromeda search engine ${ }^{82}$. The default parameters for label-free quantification (LFQ) were applied ${ }^{82}$. Matching between runs was performed, and LFQ was performed with a minimum ratio count of $2^{83}$. The iBAQ values were used to calculate the absolute protein abundance ${ }^{84,85}$

In vivo and in vitro deubiquitination assays. For in vivo deubiquitination, HEK293T cells were harvested $48 \mathrm{~h}$ after transfection with the indicated plasmids. For denaturing, lysates were heated at $95{ }^{\circ} \mathrm{C}$ for $5 \mathrm{~min}$ in the presence of $1 \%$ SDS, followed by a 10 -fold dilution with lysis buffer (to $0.1 \%$ SDS) and sonication as described in our previous publication ${ }^{60}$. The cell lysates were incubated with $S$ - 
protein or anti-MYC beads for $2 \mathrm{~h}$, and then the beads were washed with lysis buffer three times and subjected to western blot analysis with the indicated antibodies. For in vitro deubiquitination, HEK293T cells were transfected with HAubiquitin and MYC-DGCR8 plasmids; $48 \mathrm{~h}$ after transfection, MYC-DGCR8 was immunoprecipitated with anti-MYC beads and incubated with purified SFB-USP51 protein (WT or the $\mathrm{C} 372 \mathrm{~S}$ mutant) at $37^{\circ} \mathrm{C}$ for $3 \mathrm{~h}$ in deubiquitination buffer (50 mM Tris- $\mathrm{HCl}, \mathrm{pH} 8.0,50 \mathrm{mM} \mathrm{NaCl}, 10 \mathrm{mM}$ dithiothreitol, $1 \mathrm{mM}$ EDTA, 5\% glycerol). After the reaction, the beads were washed with deubiquitination buffer, and the bound proteins were eluted by boiling in $1 \times$ Laemmli buffer and subjected to western blot analysis with the indicated antibodies.

HR and NHEJ repair assays. HR and NHEJ repair assays were performed as described previously ${ }^{55}$. Briefly, LM2 cells were infected with pLCN DRR lentivirus. After G418 selection for 14 days, a single clone was established as the DRR cell line (named LM2-DRR). The LM2-DRR cells were transduced with lentivirus expressing control shRNA or shRNA targeting DGCR8, RNF168, or USP51. After puromycin selection for 2 days, the DGCR8-knockdown cells were transduced with RNAi-resistant DGCR8, S677A-DGCR8, or S677D-DGCR8, and then selected for another 7 days. The cells were co-transfected with I-SceI endonuclease (pCBA-ISceI, to induce DSBs) and pCAGGS DRR mCherry Donor EF1a BFP (an exogenous donor for HR). The cells were harvested 2 days after transfection and subjected to flow cytometric analysis (Attune NxT Flow Cytometer, Invitrogen, ThermoFisher Scientific) to determine the percentage of mCherry-positive and GFP-positive cells resulting from HR- and NHEJ-based repair, respectively. The ratio of mCherry-positive or GFP-positive cells to BFP-positive cells was used as a measure of HR or NHEJ repair efficiency. Attun ${ }^{m} \mathrm{NxT}$ software (version H.0) was used for flow cytometry data collection and analysis. A representative gating strategy for flow cytometric analysis is shown in Supplementary Fig. 11.

Clonogenic survival assay. Equal numbers of cells were plated in 6-well plates or $10-\mathrm{cm}$ tissue-culture dishes in triplicates at a clonogenic density and irradiated by using an X-RAD 320 irradiator with the indicated doses. The medium was replaced $24 \mathrm{~h}$ later and the cells were then incubated for 12-18 days. The resulting colonies were fixed and stained with crystal violet. Colonies containing more than 50 cells were counted. The survival fraction was calculated as: (number of colonies/number of cells plated $)_{\text {irradiated }} /(\text { number of colonies/number of cells plated })_{\text {non-irradiated. }}$

In vitro kinase assay. HEK293T cells were transfected with $12 \mu \mathrm{g}$ of WT FLAGATM or the kinase-dead mutant and then irradiated. Activated or kinase-dead ATM was immunopurified from the cell extracts with anti-FLAG beads (ThermoFisher Scientific, \#A36797). Recombinant MBP-tagged full-length DGCR8 or $\triangle 692-$ DGCR8, their S677A mutants, and p53 were expressed in bacteria and purified with maltose. The recombinant proteins were mixed with purified ATM protein and ATP (Cell Signaling Technology, \#9804) in kinase buffer (Cell Signaling Technology, \#9802). The samples were incubated at $30^{\circ} \mathrm{C}$ for $15 \mathrm{~min}$ in a hybridization oven-shaker (ThermoFisher Scientific). The reactions were terminated by boiling, and the proteins were subjected to western blot analysis with antibodies against p-S/TQ, p-ATM, ATM, and MBP.

Tissue microarray and immunohistochemical analysis. Human breast tumor tissue microarrays containing 139 analyzable cases of breast carcinoma were purchased from US Biolab (\#HBreD139Su01). Tissue specimens were processed as described in our previous publication ${ }^{5}$. The primary antibodies used for immunohistochemical analysis were antibodies against DGCR8 (1:250, Abcam, \#ab90579) and USP51 (1:250, Abcam, \#ab121147). Whole-slide images were captured using an Aperio CS2 slide scanner system (Leica Biosystems, Wetzlar, Germany) at a $40 \times$ magnification. Immunohistochemical staining was analyzed by using the immunoreactive score (IRS) system. The percentage of positive cells was scored on a scale of $0-4: 0$ if $0 \%$ of tumor cells were positive, 1 if $1-10 \%$ of cells were positive, 2 if $11-50 \%$ were positive, 3 if $51-80 \%$ were positive, and 4 if $81-100 \%$ were positive. Staining intensity was scored on a scale of $0-3$ ( 3 is the strongest $)$. Final IRS score $=($ score of the staining intensity $) \times($ score of the percentage of positive cells). Score $=0-6$ was considered moderate or weak expression and score $=8-12$ was considered strong expression.

Tumor radiosensitivity study. Animal experiments were performed as described previously ${ }^{86}$, in accordance with a protocol approved by the Institutional Animal Care and Use Committee of MD Anderson Cancer Center. Mice were housed at $70-74^{\circ} \mathrm{F}$ (set point: $72^{\circ} \mathrm{F}$ ) with $40-55 \%$ humidity (set point: $45 \%$ ). The light cycle of animal rooms is $12 \mathrm{~h}$ of light and $12 \mathrm{~h}$ of dark. Mice were euthanized when they met the institutional euthanasia criteria for tumor size or overall health condition. Solitary tumor xenografts were produced in the muscle of the right hind limbs of 10-week-old female nude mice (from MD Anderson Cancer Center's internal supply) by inoculation of $3 \times 10^{6}$ control, DGCR8-knockdown, or DGCR8-restored (with either WT DGCR8 or $\triangle 692$-DGCR8) LM2-R cells. Mice were randomly assigned to no treatment or treatment groups consisting of ten mice per group. Radiation treatment was initiated when tumors grew to approximately $8.0 \mathrm{~mm}$ in diameter. Fractionated doses of X-rays (2 Gy per fraction, once daily for 5 consecutive days a week $\times 2$ weeks) were delivered to the tumor-bearing limbs by using an X-RAD 320 irradiator. During irradiation, unanesthetized mice were mechanically immobilized in a jig such that the tumor was exposed in the radiation field and the animal's body was shielded from radiation exposure. Three mutually orthogonal diameters of the tumor were measured every other day with a Vernier caliper, and the mean value was calculated and used as the tumor diameter. The investigator (L. W.) who measured tumor size was blinded to the group allocation during animal experiments and outcome assessments.

Statistics and reproducibility. Each experiment was independently repeated three times, except for the animal study (one time), DUB screening (one time), tissue microarray and immunohistochemical analysis (one time), and mass spectrometric analysis (two times); the representative data are shown. Unless otherwise noted, data are presented as mean \pm SEM of three biologically independent samples. Statistical significance was determined by Student's $t$-test (two-tailed, unpaired), $\chi^{2}$ test, Pearson analysis (two-sided), or log-rank test as indicated, using GraphPad Prism 8.0 or SPSS 25.0. The statistical analysis for each plot was described in figure legends. $P<0.05$ was considered statistically significant.

Reporting summary. Further information on research design is available in the Nature Research Reporting Summary linked to this article.

\section{Data availability}

The raw data and processed data for mass spectrometric analysis of DGCR8-interacting proteins and DGCR8 phosphorylation sites have been deposited to the MassIVE database with the identifier MSV000087301. UniProt is a free public resource of protein sequence and functional information (https://www.uniprot.org/). The uncropped gels and blots are shown in Supplementary Fig. 12. Source Data are provided with this paper.

Received: 22 July 2020; Accepted: 11 June 2021;

Published online: 29 June 2021

\section{References}

1. Gianfaldoni, S. et al. An overview on radiotherapy: from its history to its current applications in dermatology. Open Access Maced. J. Med Sci. 5, 521-525 (2017)

2. Buchholz, T. A. Radiation therapy for early-stage breast cancer after breastconserving surgery. N. Engl. J. Med. 360, 63-70 (2009).

3. Jameel, J. K., Rao, V. S., Cawkwell, L. \& Drew, P. J. Radioresistance in carcinoma of the breast. Breast 13, 452-460 (2004).

4. Bao, S. et al. Glioma stem cells promote radioresistance by preferential activation of the DNA damage response. Nature 444, 756-760 (2006).

5. Zhang, P. et al. ATM-mediated stabilization of ZEB1 promotes DNA damage response and radioresistance through CHK1. Nat. Cell Biol. 16, 864-875 (2014).

6. Kirsch, D. G. et al. The future of radiobiology. J. Natl Cancer Inst. 110, 329-340 (2018).

7. Schwertman, P., Bekker-Jensen, S. \& Mailand, N. Regulation of DNA doublestrand break repair by ubiquitin and ubiquitin-like modifiers. Nat. Rev. Mol. Cell Biol. 17, 379-394 (2016).

8. Goldstein, M. \& Kastan, M. B. The DNA damage response: implications for tumor responses to radiation and chemotherapy. Annu Rev. Med 66, 129-143 (2015)

9. Ciccia, A. \& Elledge, S. J. The DNA damage response: making it safe to play with knives. Mol. Cell 40, 179-204 (2010).

10. Jackson, S. P. \& Bartek, J. The DNA-damage response in human biology and disease. Nature 461, 1071-1078 (2009).

11. Blackford, A. N. \& Jackson, S. P. ATM, ATR, and DNA-PK: the trinity at the heart of the DNA damage response. Mol. Cell 66, 801-817 (2017).

12. Price, B. D. \& D'Andrea, A. D. Chromatin remodeling at DNA double-strand breaks. Cell 152, 1344-1354 (2013).

13. Bekker-Jensen, S. \& Mailand, N. Assembly and function of DNA doublestrand break repair foci in mammalian cells. DNA Repair (Amst.) 9 , 1219-1228 (2010).

14. Wang, B. \& Elledge, S. J. Ubc13/Rnf8 ubiquitin ligases control foci formation of the Rap80/Abraxas/Brca1/Brcc36 complex in response to DNA damage. Proc. Natl Acad. Sci. USA 104, 20759-20763 (2007).

15. Sobhian, B. et al. RAP80 targets BRCA1 to specific ubiquitin structures at DNA damage sites. Science 316, 1198-1202 (2007).

16. Mailand, N. et al. RNF8 ubiquitylates histones at DNA double-strand breaks and promotes assembly of repair proteins. Cell 131, 887-900 (2007).

17. Huen, M. S. et al. RNF8 transduces the DNA-damage signal via histone ubiquitylation and checkpoint protein assembly. Cell 131, 901-914 (2007).

18. Mattiroli, F. et al. RNF168 ubiquitinates K13-15 on H2A/H2AX to drive DNA damage signaling. Cell 150, 1182-1195 (2012). 
19. Stewart, G. S. et al. The RIDDLE syndrome protein mediates a ubiquitindependent signaling cascade at sites of DNA damage. Cell 136, 420-434 (2009).

20. Doil, C. et al. RNF168 binds and amplifies ubiquitin conjugates on damaged chromosomes to allow accumulation of repair proteins. Cell 136, 435-446 (2009).

21. Kolas, N. K. et al. Orchestration of the DNA-damage response by the RNF8 ubiquitin ligase. Science 318, 1637-1640 (2007).

22. Stewart, G. S., Wang, B., Bignell, C. R., Taylor, A. M. \& Elledge, S. J. MDC1 is a mediator of the mammalian DNA damage checkpoint. Nature 421, 961-966 (2003).

23. Goldberg, M. et al. MDC1 is required for the intra-S-phase DNA damage checkpoint. Nature 421, 952-956 (2003).

24. Jackson, S. P. \& Durocher, D. Regulation of DNA damage responses by ubiquitin and SUMO. Mol. Cell 49, 795-807 (2013).

25. Lancini, C. et al. Tight regulation of ubiquitin-mediated DNA damage response by USP3 preserves the functional integrity of hematopoietic stem cells. J. Exp. Med 211, 1759-1777 (2014).

26. Mosbech, A., Lukas, C., Bekker-Jensen, S. \& Mailand, N. The deubiquitylating enzyme USP44 counteracts the DNA double-strand break response mediated by the RNF8 and RNF168 ubiquitin ligases. J. Biol. Chem. 288, 16579-16587 (2013).

27. Shanbhag, N. M., Rafalska-Metcalf, I. U., Balane-Bolivar, C., Janicki, S. M. \& Greenberg, R. A. ATM-dependent chromatin changes silence transcription in cis to DNA double-strand breaks. Cell 141, 970-981 (2010).

28. Nakada, S. et al. Non-canonical inhibition of DNA damage-dependent ubiquitination by OTUB1. Nature 466, 941-946 (2010).

29. Nicassio, F. et al. Human USP3 is a chromatin modifier required for S phase progression and genome stability. Curr. Biol. 17, 1972-1977 (2007).

30. Joo, H. Y. et al. Regulation of cell cycle progression and gene expression by H2A deubiquitination. Nature 449, 1068-1072 (2007).

31. Wang, Z. et al. USP51 deubiquitylates H2AK13,15ub and regulates DNA damage response. Genes Dev. 30, 946-959 (2016).

32. Bartel, D. P. MicroRNAs: target recognition and regulatory functions. Cell 136, 215-233 (2009).

33. Winter, J., Jung, S., Keller, S., Gregory, R. I. \& Diederichs, S. Many roads to maturity: microRNA biogenesis pathways and their regulation. Nat. Cell Biol. 11, 228-234 (2009).

34. Zhang, P. et al. miR-205 acts as a tumour radiosensitizer by targeting ZEB1 and Ubc13. Nat. Commun. 5, 5671 (2014).

35. Wang, Y. \& Taniguchi, T. MicroRNAs and DNA damage response: implications for cancer therapy. Cell Cycle 12, 32-42 (2013).

36. Korpela, E., Vesprini, D. \& Liu, S. K. MicroRNA in radiotherapy: miRage or miRador? Br. J. Cancer 112, 777-782 (2015).

37. Zhang, C. \& Peng, G. Non-coding RNAs: an emerging player in DNA damage response. Mutat. Res. Rev. Mutat. Res. 763, 202-211 (2015).

38. Majidinia, M. \& Yousefi, B. DNA damage response regulation by microRNAs as a therapeutic target in cancer. DNA Repair (Amst.) 47, 1-11 (2016).

39. Hawley, B. R., Lu, W. T., Wilczynska, A. \& Bushell, M. The emerging role of RNAs in DNA damage repair. Cell Death Differ. 24, 580-587 (2017).

40. Wei, W. et al. A role for small RNAs in DNA double-strand break repair. Cell 149, 101-112 (2012).

41. Burger, K. et al. Nuclear phosphorylated Dicer processes double-stranded RNA in response to DNA damage. J. Cell Biol. 216, 2373-2389 (2017).

42. Francia, S., Cabrini, M., Matti, V., Oldani, A. \& d'Adda di Fagagna, F. DICER, DROSHA and DNA damage response RNAs are necessary for the secondary recruitment of DNA damage response factors. J. Cell Sci. 129, 1468-1476 (2016).

43. Francia, S. et al. Site-specific DICER and DROSHA RNA products control the DNA-damage response. Nature 488, 231-235 (2012).

44. Lu, W. T. et al. Drosha drives the formation of DNA:RNA hybrids around DNA break sites to facilitate DNA repair. Nat. Commun. 9, 532 (2018).

45. Chitale, S. \& Richly, H. DICER- and MMSET-catalyzed H4K20me2 recruits the nucleotide excision repair factor XPA to DNA damage sites. J. Cell Biol. 217, 527-540 (2018)

46. Calses, P. C. et al. DGCR8 mediates repair of UV-induced DNA damage independently of RNA processing. Cell Rep. 19, 162-174 (2017).

47. Minn, A. J. et al. Genes that mediate breast cancer metastasis to lung. Nature 436, 518-524 (2005).

48. Yeom, K. H., Lee, Y., Han, J., Suh, M. R. \& Kim, V. N. Characterization of DGCR8/Pasha, the essential cofactor for Drosha in primary miRNA processing. Nucleic Acids Res. 34, 4622-4629 (2006).

49. Taneja, N. et al. Histone H2AX phosphorylation as a predictor of radiosensitivity and target for radiotherapy. J. Biol. Chem. 279, 2273-2280 (2004).

50. Olive, P. L. \& Banath, J. P. Phosphorylation of histone H2AX as a measure of radiosensitivity. Int J. Radiat. Oncol. Biol. Phys. 58, 331-335 (2004).
51. Banath, J. P., Macphail, S. H. \& Olive, P. L. Radiation sensitivity, H2AX phosphorylation, and kinetics of repair of DNA strand breaks in irradiated cervical cancer cell lines. Cancer Res 64, 7144-7149 (2004).

52. Wang, Y. et al. Gene-expression profiles to predict distant metastasis of lymph-node-negative primary breast cancer. Lancet 365, 671-679 (2005).

53. Bunting, S. F. et al. 53BP1 inhibits homologous recombination in Brcaldeficient cells by blocking resection of DNA breaks. Cell 141, 243-254 (2010).

54. Weinstock, D. M., Nakanishi, K., Helgadottir, H. R. \& Jasin, M. Assaying double-strand break repair pathway choice in mammalian cells using a targeted endonuclease or the RAG recombinase. Methods Enzymol. 409, 524-540 (2006)

55. Arnoult, N. et al. Regulation of DNA repair pathway choice in S and G2 phases by the NHEJ inhibitor CYREN. Nature 549, 548-552 (2017).

56. Lou, Z. et al. MDC1 maintains genomic stability by participating in the amplification of ATM-dependent DNA damage signals. Mol. Cell 21, 187-200 (2006).

57. Stucki, M. et al. MDC1 directly binds phosphorylated histone $\mathrm{H} 2 \mathrm{AX}$ to regulate cellular responses to DNA double-strand breaks. Cell 123, 1213-1226 (2005).

58. Bekker-Jensen, S. \& Mailand, N. The ubiquitin- and SUMO-dependent signaling response to DNA double-strand breaks. FEBS Lett. 585, 2914-2919 (2011).

59. Xiao, Z., Zhang, P. \& Ma, L. The role of deubiquitinases in breast cancer. Cancer Metastasis Rev. 35, 589-600 (2016).

60. Yao, F. et al. SKP2- and OTUD1-regulated non-proteolytic ubiquitination of YAP promotes YAP nuclear localization and activity. Nat. Commun. 9, 2269 (2018).

61. Endo, A. et al. Nucleolar structure and function are regulated by the deubiquitylating enzyme USP36. J. Cell Sci. 122, 678-686 (2009).

62. Gomez-Cabello, D. et al. Regulation of the microRNA processor DGCR8 by the tumor suppressor ING1. Cancer Res. 70, 1866-1874 (2010).

63. Zhou, Z. et al. USP51 promotes deubiquitination and stabilization of ZEB1. Am. J. Cancer Res. 7, 2020-2031 (2017).

64. Napoli, M. et al. DeltaNp63/DGCR8-dependent microRNAs mediate therapeutic efficacy of HDAC inhibitors in cancer. Cancer Cell 29, 874-888 (2016).

65. Yi, R. et al. DGCR8-dependent microRNA biogenesis is essential for skin development. Proc. Natl Acad. Sci. USA 106, 498-502 (2009).

66. Wang, Y., Medvid, R., Melton, C., Jaenisch, R. \& Blelloch, R. DGCR8 is essential for microRNA biogenesis and silencing of embryonic stem cell selfrenewal. Nat. Genet 39, 380-385 (2007).

67. Cirera-Salinas, D. et al. Noncanonical function of DGCR8 controls mESC exit from pluripotency. J. Cell Biol. 216, 355-366 (2017).

68. Deng, L. et al. Stabilizing heterochromatin by DGCR8 alleviates senescence and osteoarthritis. Nat. Commun. 10, 3329 (2019).

69. Macias, S., Cordiner, R. A., Gautier, P., Plass, M. \& Caceres, J. F. DGCR8 acts as an adaptor for the exosome complex to degrade double-stranded structured RNAs. Mol. Cell 60, 873-885 (2015)

70. Mandemaker, I. K. et al. DNA damage-induced histone $\mathrm{H} 1$ ubiquitylation is mediated by HUWE1 and stimulates the RNF8-RNF168 pathway. Sci. Rep. 7, 15353 (2017).

71. Thorslund, T. et al. Histone H1 couples initiation and amplification of ubiquitin signalling after DNA damage. Nature 527, 389-393 (2015).

72. Nowsheen, S. et al. L3MBTL2 orchestrates ubiquitin signalling by dictating the sequential recruitment of RNF8 and RNF168 after DNA damage. Nat. Cell Biol. 20, 455-464 (2018).

73. Luijsterburg, M. S. et al. A PALB2-interacting domain in RNF168 couples homologous recombination to DNA break-induced chromatin ubiquitylation. Elife 6, e20922 (2017).

74. Munoz, M. C. et al. RING finger nuclear factor RNF168 is important for defects in homologous recombination caused by loss of the breast cancer susceptibility factor BRCA1. J. Biol. Chem. 287, 40618-40628 (2012).

75. Poulsen, M., Lukas, C., Lukas, J., Bekker-Jensen, S. \& Mailand, N. Human RNF169 is a negative regulator of the ubiquitin-dependent response to DNA double-strand breaks. J. Cell Biol. 197, 189-199 (2012).

76. Zong, D. et al. BRCA1 haploinsufficiency is masked by RNF168-mediated chromatin ubiquitylation. Mol. Cell 73, 1267-1281 (2019). e1267.

77. Zhu, C. et al. SUMOylation at K707 of DGCR8 controls direct function of primary microRNA. Nucleic Acids Res 43, 7945-7960 (2015).

78. Herbert, K. M., Pimienta, G., DeGregorio, S. J., Alexandrov, A. \& Steitz, J. A. Phosphorylation of DGCR8 increases its intracellular stability and induces a progrowth miRNA profile. Cell Rep. 5, 1070-1081 (2013).

79. Ohtake, F., Tsuchiya, H., Saeki, Y. \& Tanaka, K. K63 ubiquitylation triggers proteasomal degradation by seeding branched ubiquitin chains. Proc. Natl Acad. Sci. USA 115, E1401-E1408 (2018).

80. Bosanac, I. et al. Ubiquitin binding to $\mathrm{A} 20 \mathrm{ZnF} 4$ is required for modulation of NF-kappaB signaling. Mol. Cell 40, 548-557 (2010). 
81. Nie, L. et al. The landscape of histone modifications in a high-fat diet-induced obese (DIO) mouse model. Mol. Cell Proteom. 16, 1324-1334 (2017).

82. Tyanova, S., Temu, T. \& Cox, J. The MaxQuant computational platform for mass spectrometry-based shotgun proteomics. Nat. Protoc. 11, 2301-2319 (2016).

83. Cox, J. et al. Accurate proteome-wide label-free quantification by delayed normalization and maximal peptide ratio extraction, termed MaxLFQ. Mol. Cell Proteom. 13, 2513-2526 (2014).

84. Schaab, C., Geiger, T., Stoehr, G., Cox, J. \& Mann, M. Analysis of high accuracy, quantitative proteomics data in the MaxQB database. Mol. Cell Proteom. 11, M111.014068 (2012).

85. Schwanhausser, B. et al. Global quantification of mammalian gene expression control. Nature 473, 337-342 (2011).

86. Wang, L. et al. MK-4827, a PARP-1/-2 inhibitor, strongly enhances response of human lung and breast cancer xenografts to radiation. Invest N. Drugs 30, 2113-2120 (2012).

\section{Acknowledgements}

We thank Dr Xiang Zhang (Baylor College of Medicine) for providing the LM2 cell line, Dr Sharon Dent (MD Anderson Cancer Center) for providing the anti-USP51 antibody, Dr Masayuki Komada (Tokyo Institute of Technology) for sharing the anti-USP36 antisera, and Drs Chao Wang, Mengfan Tang, Xu Feng, Jibo Wu, and Dadi Jiang (MD Anderson Cancer Center) for providing reagents and technical assistance. We thank MD Anderson's Functional Genomics Core and Characterized Cell Line Core Facility for technical support, and Christine Wogan for the critical reading of the manuscript. L.M. is supported by US National Institutes of Health (NIH) grants R01CA166051 and R01CA181029 and a Cancer Prevention and Research Institute of Texas grant RP190029. This work was also supported by an AACR Stand Up To Cancer Innovative Research Grant (award number: 403235) to L.M. and an NIH Cancer Center Support (Core) Grant P30CA016672 to The University of Texas MD Anderson Cancer Center.

\section{Author contributions}

Q.H. and L.M. conceived and designed the project. Q.H. performed and analyzed most of the experiments. L.Z. performed the DUB screening and some of the DUB experiments. L. W. and S.J.F. performed tumor radiosensitivity experiments. L.N. and J.C. performed mass spectrometric analysis. H.T. performed experiments on tissue microarrays. F.Y., Y.D., S.Y., and Y.S. contributed to reagents and protocols and provided technical assistance. Q.H. and L.M. wrote the manuscript with input from all other authors. L.M. provided scientific direction, established collaborations, and allocated funding for this study.

\section{Competing interests}

The authors declare no competing interests.

\section{Additional information}

Supplementary information The online version contains supplementary material available at https://doi.org/10.1038/s41467-021-24298-z.

Correspondence and requests for materials should be addressed to L.M.

Peer review information Nature Communications thanks Sandeep Burma and the other anonymous reviewer(s) for their contribution to the peer review of this work.

Reprints and permission information is available at http://www.nature.com/reprints

Publisher's note Springer Nature remains neutral with regard to jurisdictional claims in published maps and institutional affiliations.

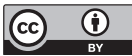

Open Access This article is licensed under a Creative Commons Attribution 4.0 International License, which permits use, sharing, adaptation, distribution and reproduction in any medium or format, as long as you give appropriate credit to the original author(s) and the source, provide a link to the Creative Commons license, and indicate if changes were made. The images or other third party material in this article are included in the article's Creative Commons license, unless indicated otherwise in a credit line to the material. If material is not included in the article's Creative Commons license and your intended use is not permitted by statutory regulation or exceeds the permitted use, you will need to obtain permission directly from the copyright holder. To view a copy of this license, visit http://creativecommons.org/ licenses/by/4.0/.

(C) The Author(s) 2021 